\title{
1 Prolonged and extended impacts of SARS-CoV-2 on the olfactory neurocircuit
}

3 Megumi Kishimoto-Urata ${ }^{1,3}$, Shinji Urata ${ }^{2,3}$, Ryoji Kagoya $^{3}$, Fumiaki Imamura ${ }^{4}$, Shin Nagayama ${ }^{5}$,

4 Rachel A. Reyna ${ }^{1}$, Junki Maruyama ${ }^{1}$, Tatsuya Yamasoba ${ }^{3}$, Kenji Kondo ${ }^{3^{*}}$, Sanae Hasegawa-Ishii ${ }^{6 *}$,

5 Slobodan Paessler ${ }^{2^{*}}$

$7 \quad{ }^{1}$ Department of Pathology, University of Texas Medical Branch, Galveston, Texas, USA

$8{ }^{2}$ Department of Otolaryngology, University of Texas Medical Branch, Galveston, Texas, USA

$9{ }^{3}$ Department of Otolaryngology, Graduate School of Medicine, the University of Tokyo, Tokyo, Japan

$10{ }^{4}$ Department of Pharmacology, Penn State College of Medicine, Hershey, Pennsylvania, USA

$11{ }^{5}$ Department of Neurobiology and Anatomy, McGovern Medical School at the University of Texas

12 Health Science Center at Houston, Texas, USA

$13{ }^{6}$ Pathology Research Team, Kyorin University, Tokyo, Japan

14

15 These authors contributed equally: Megumi Kishimoto-Urata, Shinji Urata

$16{ }^{*}$ Correspondence: kondok-tky@umin.ac.jp, sanae ishii@ks.kyorin-u.ac.jp, slpaessl@utmb.edu 


\section{Abstract}

18 The impact of SARS-CoV-2 on the olfactory pathway was studied over several time points using

19 Syrian golden hamsters. We found an incomplete recovery of the olfactory sensory neurons,

20 prolonged activation of glial cells in the olfactory bulb, and a decrease in the density of dendritic

21 spines within the hippocampus. These data may be useful for elucidating the mechanism underlying

22 long-lasting olfactory dysfunction and cognitive impairment as a post-acute COVID-19 syndrome. 


\section{Results and Discussion}

24

Severe acute respiratory syndrome coronavirus 2 (SARS-CoV-2) has infected over 215 million people, producing an average lethality of $2.1 \%$ worldwide ${ }^{1}$. Olfactory dysfunction is one of the first and most common symptoms of the coronavirus disease-2019 (COVID-19) ${ }^{2}$. The proposed mechanism underlying this SARS-CoV-2-induced olfactory dysfunction involves severe damage and impairment of the olfactory epithelium (OE); previous data using animal models indicates apoptosis and desquamation of the entire $\mathrm{OE}$, including olfactory sensory neurons $(\mathrm{OSNs})^{3}$. While the damaged OE is gradually restored in the animal study ${ }^{4}$, many COVID-19 survivors clinically continue to suffer from central nervous system (CNS) symptoms such as depression and memory impairment, as well as chronic olfactory dysfunction in some cases ${ }^{5}$. This study examined the effects of SARSCoV-2 infection on the CNS using the Syrian golden hamster model, which is a well-established model of COVID-19 that reproduces certain features of human disease . $^{6}$

Olfactory nasal cavity has a complicated structure, divided into multiple regions, e.g. medial/lateral recess on the medial-lateral axis, zone 1-4 areas on the dorsomedial-ventrolateral axis ${ }^{7}$. Each region has a different vulnerability to external harmful agents; dorsomedial side is more vulnerable to methimazole ${ }^{8}$, and lateral side is to lipopolysaccharide ${ }^{9}$. Therefore, we first analyzed the local impact of SARS-CoV-2 on the OE tentatively divided into 4 regions: dorsomedial (DM), dorsolateral (DL), ventromedial $(\mathrm{VM})$, and ventrolateral $(\mathrm{VL})$ region (Figure 1A). The hamsters were intranasally inoculated with SARS-CoV-2 at six-weeks of age and samples were collected at several time points. Using immunofluorescence, we detected a significant number of SARS-CoV-2-positive regions throughout the OE at 2 days post-infection (dpi), but not at 8 dpi (Figure 1A). Interestingly, SARSCoV-2-infected cells were observed not only superficially but also deep within portions of the DM region, including the lamina propria. The SARS-CoV-2 antigen was not observed in mature OSNs, as identified by olfactory marker protein (OMP) expression, but in cells around the OSNs, mostly supporting cells (SCs) (Figure 1B), which are known to express angiotensin converting enzyme 2 (ACE2), the receptor for SARS-CoV- $2^{10}$. The numbers of SCs significantly decreased in the VM and 
VL and could not be determined in the DM due to the complete loss of the OE at $5 \mathrm{dpi}$, although the damage was recovered almost completely in all regions by $21 \mathrm{dpi}$ (Figure 1C). Interestingly, no SARS-CoV-2 antigen was detected within the sagittal section of the whole brain (Figure 1-figure supplement $1 \mathrm{~A})$, including the olfactory bulb (OB, Figure 1-figure supplement 1B) and hippocampus (Figure 1-figure supplement 1C). The data from our study suggest that the SARSCoV-2 did not infect the brain parenchyma or that the level of infection was below detection limit, however, previous research revealed that SARS-CoV-2 RNA and viral antigen can be detected in the brain ${ }^{11}$. Moreover, we found that the OE thickness transiently decreased at $5 \mathrm{dpi}$ but recovered fully by $21 \mathrm{dpi}$, as was the case for the SC numbers (Figure1, Figure 2-figure supplement 1A,B). Nevertheless, the density of mature OSNs did not completely recover up to $42 \mathrm{dpi}$, suggesting that the maturation of OSNs may be delayed and/or incomplete (Figure 2-figure supplement 1A,C).

Our data indicate that the DM is the most vulnerable to SARS-CoV-2 in the OE. This is reasonable, as ACE2 receptors are predominantly expressed in this region ${ }^{12}$. The DM is considered to be included in the zone 1 of the zonal categorization ${ }^{7}$. Interestingly, the OSNs in zone 1 only express the endocellular enzyme $\mathrm{NAD}(\mathrm{P}) \mathrm{H}$ quinone oxido-reductase $1(\mathrm{NQO} 1)^{13}$. NQO1 acts as an antioxidant but also mediates ROS generation, being involved in increasing certain forms of neuronal damage after injury ${ }^{8}$. This led us to analyze the relationship between NQO1 expression patterns and neural damage caused by SARS-CoV-2 infection. The numbers of OMP-positive cells decreased

67 after infection and were not completely restored in both NQO1-positive and -negative regions (Figure 2A,B,C). However, the damage was more severe in NQO1-positive regions than in NQO1negative regions (Figure 2D). Furthermore, the presence of macrophages, which are labeled using the ionized calcium binding adaptor molecule 1 (lba1) suggest prolonged activation in the lamina

71 propria within NQO1-positive regions (Figure 2E,F,G). In contrast, macrophages quickly returned to

72 a normal level within NQO1-negative regions. These data suggest that NQO1-positive areas were 
next olfactory relay center, the $\mathrm{OB}$, in which the OSNs have synaptic contact with multiple neurons within the glomerulus (Figure 3A,B).

In the $\mathrm{OB}$, the density of the OSN axon terminal of each glomerulus was significantly decreased within NQO1-positive regions, but not in the NQO1-negative regions (Figure 3C,D). Interestingly, the size of the glomeruli themselves decreased not only in NQO1-positive but also in NQO1-negative regions (Figure 3E,F). These data indicate that SARS-CoV-2 infection impacts odor information processing within the whole OB, but especially prominent in NQO1-positive regions. Next, we carefully examined the effects of SARS-CoV-2 on the profile of glial activities (Figure 4A, Figure 4figure supplemental 1A, Figure 5-figure supplement $1 \mathrm{~A}$ ) throughout the multiple layers of the OB: the olfactory nerve layer (ONL) where the OSN axon shaft are densely packed, the glomerular layer $(\mathrm{GL})$ where the OSN axon synapse to the relay neurons, and the external plexiform layer (EPL) where the projection neurons interact with the local interneurons. Remarkably, the lba1 signal peaked at $17 \mathrm{dpi}$ (Figure 4B), in contrast to the OE, in which it peaked at $5 \mathrm{dpi}$, indicating that microglia in the OB became activated more slowly than macrophages in the OE. Interestingly, the ONL, occupied by OSN axons, showed significantly strong microglial activation, even at 5 dpi. This indicates that the microglia in the ONL respond to the damage of the OSN axons from the OE quickly and directly, and this damage would provide an indirect secondary impact on the postsynaptic circuits in the GL and EPL. Consistent with the impact of SARS-CoV-2 on the OSN axon terminals within the OB (Figure 3), the impact on the NQO1-positive regions was significantly

93 stronger within the ONL at $5 \mathrm{dpi}$ and within the GL at $17 \mathrm{dpi}$, as compared to that of the NQO-1-

94 negative regions (Figure 4B,D,F). However, the area occupied by glial fibrillary acidic protein

95 (GFAP)-expressing reactivated astrocytes was enlarged at $17 \mathrm{dpi}$ within the GL (Figure 4-figure

96 supplemental 1B,C,E), although the dynamics of GFAP-positive cells were not different based on

97 NQO1 expression (Figure 4-figure supplemental 1D,F). This indicates that astrocyte activity is

98 strongly associated with the activity of microglia in the GL compared to that in other layers. 
Next, we examined the impact of SARS-CoV-2 on higher brain areas, including the piriform cortex (PC) and the hippocampus (Figure 5, Figure 5-figure supplemental 1). In the PC, which is one of the olfactory cortices receiving direct inputs from the OB, GFAP-expression appeared transient in the pia mater but persisted in the layer 1 of the PC up to 42 dpi (Figure 5-figure supplemental 1B). Activated microglia/macrophages cells labeled by lba1 within the pia mater increased in numbers at 5 and $17 \mathrm{dpi}$, although the lba1-positive area was unchanged in layer 1 (Figure 5-figure supplemental 1C,D). The peak of this activity was not correlated to the presence of SARS-CoV-2 within the nasal cavity (Figure 1A), indicating that glial activation in the PC may not be induced directly by the virus through the olfactory pathway but by local and/or systemic inflammatory responses. In fact, inflammatory cytokines are systemically elevated in both severe and non-lethal COVID-19 models $^{14}$. Given that ACE2 is expressed in the Bowman's glands of the lamina propria ${ }^{15}$ and that macrophages there were activated after SARS-CoV-2 infection (Figure 2F,G), the immune

111 response in the lamina propria may induce the elevation of intravascular inflammatory cytokines,

112 triggering the activation of glial cells and macrophages in the CNS. In the hippocampus, GFAP-

113 positive astrocytic endfeet were easily detectable around the blood vessels of the apical dendritic

114 region at 5, 8, 17, and 42 dpi (Figure 5B), as compared to those of the basal region. The peak Iba1115 signal in the basal region was achieved by $8 \mathrm{dpi}$, with the area being restored by $42 \mathrm{dpi}$ (Figure $1165 \mathrm{C}, \mathrm{D})$. In contrast, the Iba1-signal in the apical region remained strong until 42 dpi (Figure 5C,D,E).

117 These data indicate that SARS-CoV-2 infection in the nostril triggered the activation of microglia and 118 astrocytes, even in the hippocampus, and that the impacts are significantly different in individual 119 layer. Thus, an interesting question could be posed as to whether the activated microglia and 120 astrocytes could induce any changes in the neuronal circuits?

121 There are many reports that reveal glial cells induce synaptic modulation ${ }^{16}$, synaptic loss ${ }^{9}$, synaptic 122 plasticity ${ }^{17}$, and change of synaptic density ${ }^{18}$. These changes may be associated with dementia ${ }^{19}$.

123 Therefore, we analyzed dynamics of spine density within the hippocampus using Golgi stained brain 124 sections (Figure 5F,G). Basal dendritic spines in the hippocampal CA1 were extremely stable. In 
125 contrast, the density of apical dendritic spines was significantly decreased at 42 dpi (Figure $5 \mathrm{H}$ ) This

126 finding may be associated with the prolonged activation of microglial cells, most notably significant at

$12742 \mathrm{dpi}$ (Figure 5E). These results suggest that intranasal inoculation of SARS-CoV-2 induces glial

128 cell activation and changes spine density within the higher brain regions, including the

129 hippocampus. Future studies should determine whether these changes impact animal behavior and

130 if so, how long it is required for recovery.

131 In summary, we report that single priming with SARS-CoV-2 resulted in long-lasting effects in

132 hamsters, not only on the OE but also within the brain regions critical for cognitive function. We

133 found drastic CNS changes including glial activation and synaptic dynamics, without any SARS-

134 CoV-2 antigen by immunostaining. Our study may be of importance for better understanding the

135 mechanism(s) driving the olfactory impairment and potentially cognitive dysfunction present in

136 COVID-19 survivors. 


\section{References}

139 1. Johns Hopkins University and Medicine, Coronavirus Resouce Center. Available from:

$140 \quad$ https://coronavirus.jhu.edu.

141 2. Gandhi, R. T., Lynch, J. B. \& del Rio, C. Mild or Moderate Covid-19. N. Engl. J. Med. 1-9 (2020).

142 3. Ye, Q. et al. SARS-CoV-2 infection in the mouse olfactory system. Cell Discov. 7, 1-13 (2021).

143 4. Urata, S. et al. Regeneration Profiles of Olfactory Epithelium after SARS-CoV-2 Infection in

144 Golden Syrian Hamsters. ACS Chem. Neurosci. (2021).

145 5. Kumar, S., Veldhuis, A. \& Malhotra, T. Neuropsychiatric and Cognitive Sequelae of COVID-19.

$146 \quad$ Front. Psychol. 12, 1-6 (2021).

147 6. Sia, S. F. et al. Pathogenesis and transmission of SARS-CoV-2 in golden hamsters. Nature 583, $148 \quad$ 834-838 (2020).

7. Buck, L. \& Axel, R. A novel multigene family may encode odorant receptors: a molecular basis for odor recognition. Cell 65, 175-187 (1991).

8. Tuerdi, A. et al. Dorsal-zone-specific reduction of sensory neuron density in the olfactory epithelium following long-term exercise or caloric restriction. Sci. Rep. 8, 1-16 (2018).

9. Hasegawa-Ishii, S., Shimada, A. \& Imamura, F. Lipopolysaccharide-initiated persistent rhinitis causes gliosis and synaptic loss in the olfactory bulb. Sci. Rep. 7, 1-14 (2017).

10. Brann, D. H. et al. Non-neuronal expression of SARS-CoV-2 entry genes in the olfaory system suggests mechanisms underlying COVID-19-associated anosmia. Sci. Adv. 5801, eabc5801 (2020).

11. de Melo, G. D. et al. COVID-19-related anosmia is associated with viral persistence and inflammation in human olfactory epithelium and brain infection in hamsters. Sci. Transl. Med. 13, 
eabf8396 (2021).

161 12. Fodoulian, L. et al. SARS-CoV-2 Receptors and Entry Genes Are Expressed in the Human Olfactory Neuroepithelium and Brain. iScience 23, (2020).

13. Gussing, F. \& Bohm, S. NQO1 activity in the main and the accessory olfactory systems correlates with the zonal topography of projection maps. Eur. J. Neurosci. 19, 2511-2518 (2004).

14. Jiao, L. et al. The olfactory route is a potential way for SARS-CoV-2 to invade the central nervous system of rhesus monkeys. Signal Transduct. Target. Ther. 6, 169 (2021).

15. Chen, M. et al. Elevated ACE-2 expression in the olfactory neuroepithelium: Implications for anosmia and upper respiratory SARS-CoV-2 entry and replication. Eur. Respir. J. 56, (2020).

16. Schafer, D. P. et al. Microglia Sculpt Postnatal Neural Circuits in an Activity and ComplementDependent Manner. Neuron 74, 691-705 (2012).

171 17. Abbott, L. F. \& Nelson, S. B. Synaptic plasticity: taming the beast. Nat. Neurosci. 3, 1178-1183 (2000).

18. Zhan, Y. et al. Deficient neuron-microglia signaling results in impaired functional brain connectivity and social behavior. Nat. Neurosci. 17, 400-406 (2014).

19. Clare, R., King, V. G., Wirenfeldt, M. \& Vinters, H. V. Synapse loss in dementias. J. Neurosci. Res. 88, 2083-2090 (2010).

177 20. Jiao, Y. et al. A simple and sensitive antigen retrieval method for free-floating and slide-mounted tissue sections. J. Neurosci. Methods 93, 149-162 (1999).

179 21. Ranjan, A. \& Mallick, B. N. Differential staining of glia and neurons by modified Golgi-Cox method. J. Neurosci. Methods 209, 269-279 (2012).

181 22. Angulo, A., Merchan, J. A. \& Molina, M. Golgi-Colonnier method: Correlation of the degree of 

(1994).

184

185

186

187

188

189

190

191

192

23. Berbel, P. J. Chromation at low temperatures improves impregnation of neurons in Golgi-aldehyde methods. J. Neurosci. Methods 17, 255-259 (1986).

24. Tokuno, H., Nakamura, Y., Kudo, M. \& Kitao, Y. Effect of Triton X-100 in the Golgi-Kopsch method. J. Neurosci. Methods 35, 75-77 (1990).

25. Ranjan, A. \& Mallick, B. N. A modified method for consistent and reliable Golgi-Cox staining in significantly reduced time. Front. Neurol. DEC, 1-8 (2010).

26. Kikuta, S. et al. Sensory deprivation disrupts homeostatic regeneration of newly generated olfactory sensory neurons after injury in adult mice. J. Neurosci. 35, 2657-2673 (2015).

27. SMITH, O. A. J. \& BODEMER, C. N. A stereotaxic atlas of the brain of the golden hamster (Mesocricetus auratus). J. Comp. Neurol. 120, 53-63 (1963).

28. Knafo, S., Ariav, G., Barkai, E. \& Libersat, F. Olfactory learning-induced increase in spine density along the apical dendrites of CA1 hippocampal neurons. Hippocampus 14, 819-825 (2004).

29. Mitra, R., Jadhav, S., McEwen, B. S., Vyas, A. \& Chattarji, S. Stress duration modulates the spatiotemporal patterns of spine formation in the basolateral amygdala. Proc. Natl. Acad. Sci. U. S. A. 102, 9371-9376 (2005). 
A, Distribution of SARS-CoV-2 in the olfactory epithelium (OE) at mock, $2 \mathrm{dpi}$, and $8 \mathrm{dpi}$. The OE was divided into 4 regions: dorsomedial (DM), dorsolateral (DL), ventromedial (VM), and ventrolateral (VL). SARS-CoV-2-positive cells were localized in the 4 regions of the OE at 2 dpi. The white arrows indicate the SARS-CoV-2-positive cells in the lamina propria. Scale bars, $1 \mathrm{~mm}$ (left), $100 \mu \mathrm{m}$ (right). $\mu \mathrm{m}$.

211 followed by Dunnett's post hoc test. ${ }^{*} p<0.05$, ${ }^{* *} p<0.01,{ }^{* *} p<0.001, \dagger$ means unevaluable as the

212 OE was completely desquamated and SCs were not detectable). Number of samples; $\mathrm{n}=3(5,21$

213 and $42 \mathrm{dpi}$ ) and 4 (mock).

Figure 2 | Region-specific damage of the olfactory epithelium (OE) due to SARS-CoV-2 infection.

A, Representative coronal section of the OE stained with olfactory marker protein (OMP), NAD(P)H

217 quinone oxido-reductase 1 (NQO1), and DAPI. The white dashed line is the border of the NQO1-

218 positive or -negative region. Scale bar, $1 \mathrm{~mm}$. epithelium. Scale bar, $100 \mu \mathrm{m}$. 
222 C, Numbers of OMP-positive cells in the NQO1-positive or -negative region. (one-way ANOVA

223 followed by Dunnett's post hoc test. ${ }^{* *} p<0.01$, ${ }^{* * *} p<0.001$, $\uparrow$ means unevaluable as the OE was

224 completely desquamated and OMP-positive cells were not countable). Number of samples: $n=3(5$,

22521 and 42 dpi) and 4 (mock).

226 D, Comparison of the normalized density of OMP-positive cells in NQO1-positive and NQO1-

227 negative regions. $\left(n=3\right.$, Welch's t-test. ${ }^{*} p<0.05, \dagger$ means unevaluable as the OE was completely

228 desquamated and OMP-positive cells were not countable).

229 E, Representative coronal section of the OE stained with ionized calcium binding adaptor molecule 1

230 (Iba1), NQO1, and DAPI. The white dashed line is the border of the NQO1-positive or -negative

231 region. Scale bar, $1 \mathrm{~mm}$.

232 F, Representative images of Iba1-positive cells at 5, 17, and 42 dpi or mock. The white dashed line

233 is the border between the OE and lamina propria. Lamina propria, LP. Scale bar, $100 \mu \mathrm{m}$.

234 G, Densities of Iba1-positive cells in the NQO1-positive or -negative region. (one-way ANOVA

235 followed by Dunnett's post hoc test. $\left.{ }^{* * *} p<0.001\right)$. Number of samples: $n=3(5,17$ and $42 \mathrm{dpi})$ and 2364 (mock).

238 Figure 3 | Region-specific changes in the glomeruli in the olfactory bulb (OB) due to SARS-CoV-2 239 infection.

240 A, Representative coronal section of the OB stained with olfactory marker protein (OMP), NQO1, 241 and DAPI. Scale bar, $1 \mathrm{~mm}$.

242 B, Representative time series of glomeruli stained with OMP. Each circled area corresponds to a 243 glomerulus. Scale bar, $100 \mu \mathrm{m}$. 
244 C, The density of OMP-positive areas within the glomerulus compared between NQO1-positive or -

245 negative regions. ( $n=3$, one-way ANOVA followed by Dunnett's post hoc test. ${ }^{* *} p<0.01$, ${ }^{* * *} p<$

246 0.001).

247 D, Comparison of the normalized OMP-positive area in NQO1-positive and NQO1-negative regions.

$248 \quad\left(n=3\right.$, Welch's t-test. $\left.{ }^{* *} p<0.01\right)$.

$249 \mathrm{E}$, The size of the glomerulus at $42 \mathrm{dpi}$ and mock. (Welch's t-test. ${ }^{* *} \mathrm{p}<0.001$ ). Number of samples;

$250 \mathrm{n}=78,60,86$, and 67 glomeruli from 42 dpi of NQO1-positive, mock of NQO1-positive, 42 dpi of

251 NQO1-negative, and mock of NQO1-negative, $n=3$ animals in individual group.

$252 \mathrm{~F}$, Histogram of the area of the glomerulus. The regression curves were fitted using kernel density 253 estimation.

255 Figure 4 | Region-specific microglial activation in the OB due to SARS-CoV-2 infection.

256 A, Representative coronal section of the OB stained with Iba1, NQO1, and DAPI. The OB has a

257 multi-layered structure from surface to the center; Olfactory nerve layer (ONL), Glomerular layer (GL),

258 External plexiform layer (EPL), Mitral cell layer (MCL), Internal plexiform layer (IPL), and Granule cell

259 layer (GNL). Squares are representative regions in the analysis of NQO1-positive and -negative

260 region. Scale bar, $1 \mathrm{~mm}$.

261 B, Representative images of ONL, GL, and EPL stained with Iba1, NQO1, and DAPI. White dashed

262 circles indicate glomeruli. Scale bar, $200 \mu \mathrm{m}$.

263 C,E,G, Iba-1-positive area in the ONL (c), GL (e), and EPL (g). ( $n=3$, one-way ANOVA followed by

264 Dunnett's post hoc test. $\left.{ }^{*} p<0.05,{ }^{* *} p<0.01,{ }^{* * *} p<0.001\right)$. 
$\mathrm{D}, \mathrm{F}, \mathrm{H}$, Comparison of the normalized values of the Iba1-positive area in NQO1-positive and NQO1negative regions in the ONL (d), GL (f), and EPL (h). $\left(n=3\right.$, Welch's t-test. $\left.{ }^{*} p<0.05,{ }^{* *} p<0.01\right)$.

Figure 5 | Glial activation and loss of dendritic spines in the hippocampus due to SARS-CoV-2 infection.

A, The upper image is a representative coronal image of the cerebral hemisphere stained with lba1,

271 GFAP, and DAPI. The upper white square indicates the hippocampal formation (HPF). The lower

272 panel is a high-magnification image. The lower white square-labeled region (CA1) is enlarged in

273 Figure 5B. Scale bar, $5 \mathrm{~mm}$ (upper), $1 \mathrm{~mm}$ (lower).

$\mathrm{B}$, Representative image of astrocytes in the CA1 stained with glial fibrillary acidic protein (GFAP) and DAPI at 5, 8, 17, 42 dpi, and mock. Arrows indicate GFAP-positive astrocytic endfeet. Corpus

277 lacunosum-moleculare, slm. Scale bar, $100 \mu \mathrm{m}$

279 Arrows indicate lba1-positive microglia. Scale bar, $100 \mu \mathrm{m}$ followed by Dunnett's post hoc test. $\left.{ }^{*} p<0.05,{ }^{* *} p<0.01,{ }^{* *} p<0.001\right)$.

282 E, Comparison of the normalized density of lba1-positive cells in the NQO1-positive and NQO1283 negative regions. $\left(n=3\right.$, Welch's t-test. $\left.{ }^{*} p<0.05,{ }^{* *} p<0.01\right)$.

$284 \mathrm{~F}$, Upper panel is a representative image depicting Golgi-impregnated neurons of the cerebral 285 hemisphere. Lower panel is a high-magnification image of the CA1. Scale bar, $100 \mu \mathrm{m}$. 
286 G, Representative image depicting Golgi-impregnated dendrites and dendritic spines in the apical

287 and basal region of the CA1. Scale bar, $5 \mu \mathrm{m}$.

$288 \mathrm{H}$, Densities of apical and basal dendritic spines. ( $n=3$, one-way ANOVA followed by Tukey's post

289 hoc test. ${ }^{*} p<0.05,{ }^{* *} p<0.01$, neurons $=24,48,46$; spines $=1610,1789,2453$ from the basal

290 dendrites of $8,42 \mathrm{dpi}$, and mock group, neurons $=25,41,43$; spines $=1252,1262,1317$ from the

291 apical dendrites of 8, 42 dpi and mock group) . 


\section{Methods}

Cell and virus

Vero E6 cells were maintained using Dulbecco's modified Eagle's medium (DMEM) supplemented with $10 \%$ fetal bovine serum (FBS), $1 \%$ penicillin-streptomycin, and L-glutamine. SARS-CoV-2 (USA/WA-1/2020) was propagated in Vero E6 cells with DMEM supplemented with $2 \%$ FBS. Cell culture supernatant was stored in the $-80^{\circ} \mathrm{C}$ freezer until use.

Animal experiments

Six-week-old female Syrian golden hamsters were purchased from Charles River. Syrian golden hamsters were anesthetized with isoflurane and inoculated bilateral-intranasally with $10^{5} 50 \%$ tissue culture infectious dose (TCID50) of SARS-CoV-2 diluted in $100 \mu \mathrm{l}$ of phosphate-buffered saline (PBS) or PBS as a mock control. Mock controls were sacrificed 42 days after inoculation. All hamsters were housed in the animal biosafety level-2 (ABSL-2) and ABSL-3 facilities within the Galveston National Laboratory at the University of Texas Medical Branch (UTMB). All animal studies are reviewed and approved by the Institutional Animal Care and Use Committee at UTMB and are conducted according to the National Institutes of Health guidelines.

311 to collect samples. The heads of hamsters were fixed in $10 \%$ buffered formalin for 7 days before

312 removal from the BSL-3 facility and followed by incubation in phosphate buffered saline (PBS). After 313 OBs were extracted from the skull, right OBs were sectioned into $100 \mu \mathrm{m}$ thick sections using a 314 vibratome and left OBs were proceeded to the tissue Golgi-Cox procedures. The nasal tissues 
315 including the olfactory epithelium were decalcified with $10 \%$ EDTA (pH 7.0) for 7 days at $37^{\circ} \mathrm{C}$ with

316 gentle shaking. Decalcification samples were dehydrated in $100 \%$ ethanol three times and

317 embedded in paraffin. Serial coronal sections (5 $\mu \mathrm{m}$ thick) were cut and mounted on glass slides.

318 Deparaffinized sections and free-floating brain slices were incubated for 10-30 min in Target

319 Retrieval Solution (S1700; Dako) at $72-102{ }^{\circ} \mathrm{C}$ in a water-bath for antigen retrieval ${ }^{20}$. Non-specific

320 antibody binding was blocked by Serum-free protein block (Dako). Samples were incubated for $12 \mathrm{hr}$

321 in a solution containing the following primary antibodies: olfactory marker protein (OMP, goat

322 polyclonal, 1:5000 dilution; Wako Chemicals), SARS-CoV-2 Nucleocapsid antibody (rabbit polyclonal,

323 1:100; Sino Biological), anti-lba1 (rabbit polyclonal, 1:300; Wako), anti-lba1 for paraffin section

324 (rabbit polyclonal, 1:300; Wako), anti-lba1 (goat polyclonal, 1:300; Abcam), anti-NQO1 (rabbit

325 polyclonal, 1:300; Cell Signaling), anti-NQO1 (goat polyclonal, 1:300; Abcam), and anti-GFAP

326 antibody (mouse monoclonal, 1:500; Millipore). Unbound antibodies were removed by washing for 3

327 min with PBS or 30 min with PBS containing $0.1 \%$ Triton X-100, respectively. Primary antibodies

328 were detected by incubation for $1 \mathrm{hr}$ with a solution containing the following secondary antibodies;

329 goat anti-rabbit Fluor 488, goat anti-mouse Fluor 568, donkey anti-goat Alexa Fluor 488, and donkey

330 anti-rabbit Alexa Fluor 568 (1:200; Invitrogen). To visualize cell nuclei, DAPI or Hoechst 33342

331 (1:500; Thermo Fisher) was applied during the secondary antibody stain. Samples were mounted in

332 Vectashield hard-set mounting medium (Vector Laboratories) or 70\% Glycerol.

Golgi-Cox staining

335 To quantify the number of dendritic spines, brain slices were treated with the Rapid GolgiStain Kit 336 according to the manufacturer's instructions (ND Neurotechnologies, Inc., PK401) with minor

337 modifications. Since sample conditions, especially after formalin fixation, were significant and critical 338 variables for Golgi staining ${ }^{21-24}$, modified Golgi-Cox staining was performed as described in previous 339 reports ${ }^{24,25}$. Briefly, the brain slices were incubated in the impregnation solution for 14 days in $37^{\circ} \mathrm{C}$. 
The impregnation solution was mixed using a 1:1 ratio of Solutions $A$ and $B$, which contain mercuric chloride, potassium dichromate, and potassium chromate. The solution was prepared $24 \mathrm{hr}$ before use, to allow precipitate formation, and covered with aluminum foil and kept in $37^{\circ} \mathrm{C}$. The sample was replaced in a sucrose-based solution (solution $\mathrm{C}$ ) for $10 \mathrm{~min}$ at room temperature. The sections were transferred and agglutinated onto gelatin-coated slides. The sections were rinsed twice for 5 min each in distilled water to remove the impregnation solution. The sections were then incubated in the staining solution (Solution D: Solution E: distilled water, 1:1:2) for $10 \mathrm{~min}$ at room temperature.

Analysis of immunohistochemistry.

To minimize the anatomical variations of the nose, three coronal sections covered with olfactory neuroepithelium were analyzed every $500 \mu \mathrm{m}$ intervals. The OE consists of three cell types: olfactory sensory neurons, supporting cells, and basal stem cells. For each OE, coronal sections of the OE were divided into 4 regions: dorsomedial (DM), dorsolateral (DL), ventromedial (VM) and ventrolateral $(\mathrm{VL})$. The thickness of olfactory epithelium was measured between the surface of neuroepithelium and the line of basal cells. We counted the numbers of OMP-positive cells for mature olfactory sensory neurons. To evaluate the numbers of supporting cells (SCs), we defined and counted the SCs as the columnar as the cells located proximal to the nasal cavity, as

357 indicated in a previous report ${ }^{26}$. To investigate the presence of macrophages in the lamina propria, Iba1-positive cells were counted. All analyses were performed for each group per $100 \mu \mathrm{m}$ OE length.

359 Three regions were randomly selected, with the regions being at least $100 \mu \mathrm{m}$ apart, and the 360 averaged values were used. To quantify the densities of the Iba1- and GFAP-positive area, we 361 defined the positive area as one that exceeds two SDs of the mean background intensity.

362 Subsequently, binary images were overlapped onto the original images and the densities were 363 automatically measured using ImageJ software. OMP-positive cells (Figure 2D), Iba1-postive areas 
supplement 1D,F) were adjusted to normalize the average values in the NQO1-negative. Iba1positive areas in the hippocampus (Figure 5E) were adjusted to normalize the values in the basal region.

Analysis of Dendritic Spine Density

The location of CA1 was identified using a previously published brain map ${ }^{27}$. Pyramidal neurons in the CA1 were selected and three apical and three basal dendritic segments were analyzed ${ }^{28}$. For analysis of Golgi-impregnated neurons, the criteria are the following, according to a previous report $^{29}$ : (1) dendrites with a length of at least $50 \mu \mathrm{m}$, (2) consistent and dark impregnation along the entire extent of dendrites, and (3) relative isolation from neighboring impregnated dendrites.

Densities of dendritic spines were calculated as the mean numbers of spines per $10 \mu \mathrm{m}$ per dendrite per neuron in individual animals per group.

\section{Microscopy}

Images (Figure 5F,G, Figure 1-figure supplement 1A, and Figure 2-figure supplement 1A) were captured using a digital CCD (Keyence BZ-X800, Japan) with $20 \times(\mathrm{NA}=0.45), 100 \times(\mathrm{NA}=1.45)$ objective lenses. The size of $20 \times$ single horizontal images (Figure 5F, Figure 1-figure supplement $1 \mathrm{~A}$, and Figure 2-figure supplement $1 \mathrm{~A}$ ) were set to $1920 \times 1440$, with pixel size of $0.37 \times 0.37$ and z-spacing of $5 \mu \mathrm{m}$. The size of $100 \times$ single horizontal images (Figure $5 \mathrm{G}$ ) were set to $1920 \times$ 1440 , with pixel size of $0.07 \times 0.07$ and $z$-spacing of $0.2 \mu \mathrm{m}$. For samples labeled with multiple antibodies (Figure 1A,B, Figure 2A,B,E,F, Figure 3A,B, Figure 4A,B, Figure 5A,B,C, Figure 1figure supplement 1B,C, Figure 2-figure supplement 1A, Figure 4-figure supplement 1A,B, and Figure 5-figure supplement $1 \mathrm{~A}, \mathrm{~B})$, images were obtained using a confocal microscope (Zeiss LSM 880 , Germany) with a 20 objective lens (NA = 0.8). Fluorophores were excited by 405, 488, $561 \mathrm{~nm}$ 
lines of diode lasers. The sizes of single horizontal images were set to $512 \times 512$, with pixel size of $0.83 \times 0.83$ and z-spacing of $1 \mu \mathrm{m}$.

To prevent arbitrary analysis, all data were randomly and blindly analyzed by two trained human operators. R (https://www.r-project.org/) and custom-written Python scripts were used for statistical analysis. Mean \pm SD was used to indicate biological variations. In Figure2D, Figure 3D,E, Figure 4D, F, H, Figure 5E, Figure 4-figure supplemental 1D, and Figure 4-figure supplemental 1F, Welch's ttests were used. Compared to mock groups, comparisons among multiple groups (Figure 1C, Figure 2C,G, Figure 3C,E, Figure 5D, Figure 2-figure supplement B,C, Figure 4-figure supplemental C,E,

399 and Figure 5-figure supplement 1C,D) were evaluated with one-way ANOVA and followed by

400 Dunnett's post hoc test. In Figure 5H, one-way ANOVA and followed by Tukey's post hoc test were 401 performed. P-values for figures are depicted as follows: ${ }^{*} p<0.05,{ }^{* *} p<0.01,{ }^{* *} p<0.001$.

404 We would like to thank both the histology core for the HE-staining and imaging core for use of the 405 microscope at UTMB, C.A. Grant, M.A. Micci, K. Suenaga, and H. ligusa for technical support in 406 preparation of the manuscript, as well as R. Kamei T.Higashi and Y. Kashiwagi for discussions. We 407 also thank T. Makishima for critical reading of the manuscript. This project was funded by John 408 S. Dunn Endowment to S.P. 
411 J.M. designed the study. R.A.R. and J.M. collected the samples. M.K.U. and S.U. designed the

412 experiments and performed the experiments and imaging. M.K.U, S.U., R.K., and K.K. quantified

413 and M.K.U, S.U., and S.H.I. analyzed data. M.K.U, S.U., R.K., K.K., S.H.I, F.I., S.N., T.Y., and S.P.

414 interpreted data. S.U. designed the statistical analysis. S.H.I., F.I., S.N., R.K., T.Y., K.K. and S.P

415 supervised the experiments. M.K.U. and S.U. co-wrote the initial manuscript. S.H.I., F.I., S.N., R.K.,

416 K.K., J.M., R.A.R., T.M., T.Y. and S.P. revised the manuscript. S.P. conceived the study, designed

417 experiments, interpreted data, and co-wrote the manuscript with input from all authors. 
Figure supplements legends.

Figure 1-figure supplement 1 | Distribution of SARS-CoV-2 in the OB.

A, Sagittal sections of the whole brain stained by SARS-CoV-2 in mock and 2 dpi. Planes A and B

421 are shown as coronal sections in the Figure B and C. Scale bar, $1 \mathrm{~cm}$.

423 images of the GL in NAD(P)H quinone oxido-reductase 1 (NQO1)-positive (upper) and -negative

424 (lower) regions. White dashed circles indicate glomeruli. Scale bars, $1 \mathrm{~mm}$ (left), $100 \mu \mathrm{m}$ (right). white box indicates hippocampal formation (HPF). Right panel is a high-magnification image of the HPF; CA1, CA3, and DG. Scale bar, $1 \mathrm{~mm}$. mock,. Scale bars, $1 \mathrm{~mm}$ (left), $100 \mu \mathrm{m}$ (right).

434 Number of samples; $n=3$ (21 dpi) and 4 (5, 42 dpi, and mock). 
Figure 4-figure supplement 1 | GFAP-positive cells in the OB.

441 A, Representative coronal section of the OB stained with glial fibrillary acidic protein (GFAP), NQO1, 442 and Hoechst. Scale bar, $1 \mathrm{~mm}$.

$443 \mathrm{~B}$, Representative images of the olfactory nerve layer (ONL), glomerular layer (GL), and external 444 plexiform layer (EPL) stained with GFAP, NQO1, and DAPI. White dashed circles indicate glomeruli. 445 Scale bar, $200 \mu \mathrm{m}$.

446 C,E, Areas of GFAP-positive cells in the ONL and GL. $(n=3$, one-way ANOVA followed by

447 Dunnett's post hoc test. $\left.{ }^{* *} p<0.01,{ }^{* * *} p<0.001\right)$.

448 D,F, Comparison of the normalized values of the density of GFAP-positive cells between NQO1-

449 positive and NQO1-negative regions in the ONL and GL. ( $n=3$, Welch's t-test).

Figure 5-figure supplement 1/ Glial activities in the piriform cortex (PC) due to SARS-CoV-2 infection.

452 A, Representative coronal image of the cerebral hemisphere stained with ionized calcium binding 453 adaptor molecule 1 (Iba1), GFAP, and Hoechst. The white arrow indicates the PC and the white 454 dashed square is a region of interest in the study. Scale bar, $1 \mathrm{~mm}$.

455 B, Representative images of the layers of pia mater, layer 1 (L1), and layer 2 (L2) stained with Iba1, 456 GFAP, and Hoechst at 5, 17, and 42 dpi and mock. White and yellow arrows show Iba1- and GFAP457 positive cells, respectively. Scale bar, $100 \mu \mathrm{m}$.

458 C, Quantitative analysis of Iba1-positive cells in the pia mater. ( $n=3$, one-way ANOVA followed by 459 Dunnett's post hoc test. $\left.{ }^{*} p<0.05,{ }^{* *} p<0.01\right)$. 
bioRxiv preprint doi: https://doi.org/10.1101/2021.11.04.467274; this version posted November 5, 2021. The copyright holder for this preprint (which was not certified by peer review) is the author/funder, who has granted bioRxiv a license to display the preprint in perpetuity. It is made

A available under aCC-BY 4.0 International license.

\section{SARS-CoV-2 DAPI}
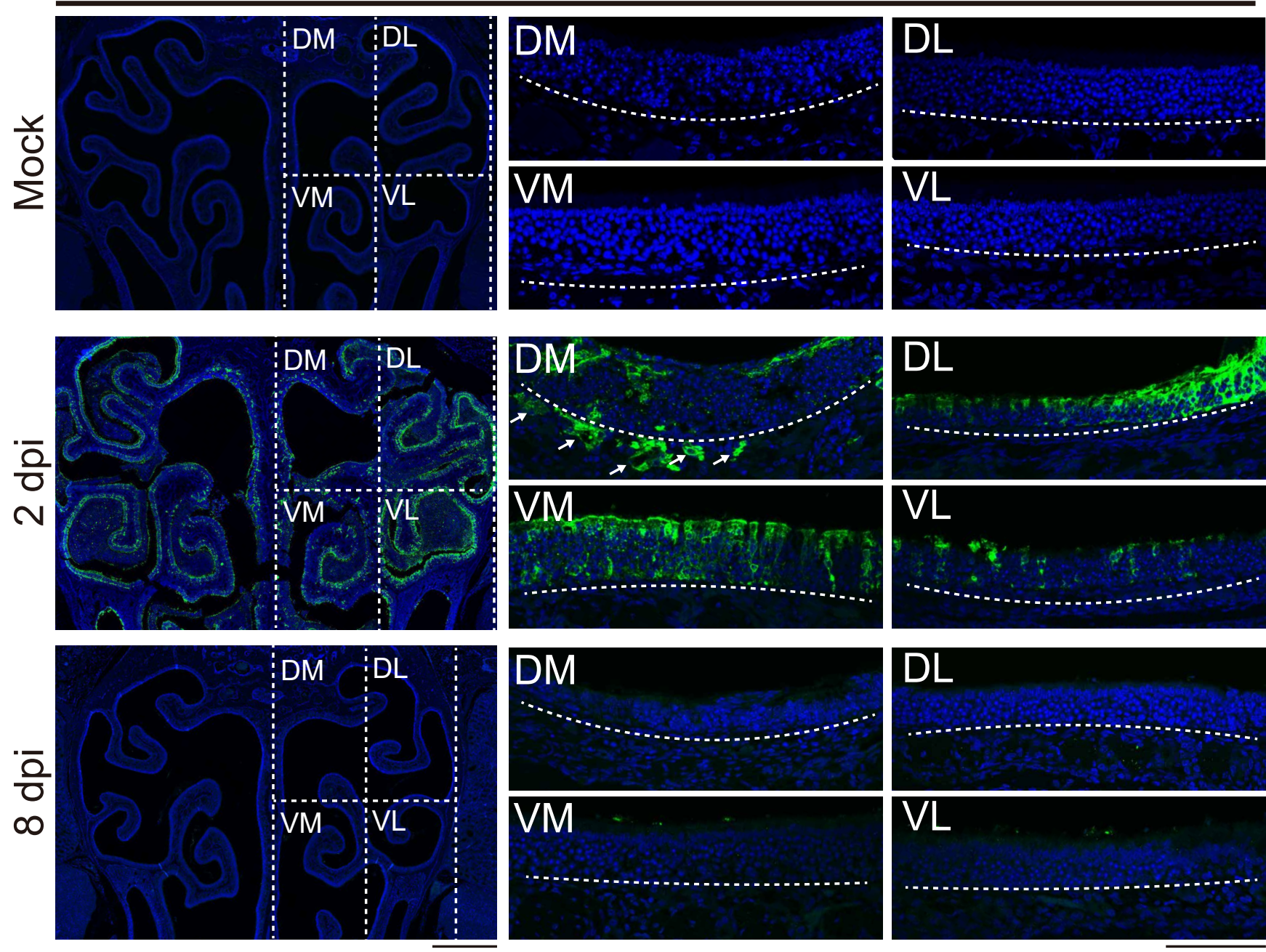

\section{$\mathrm{DL}$}
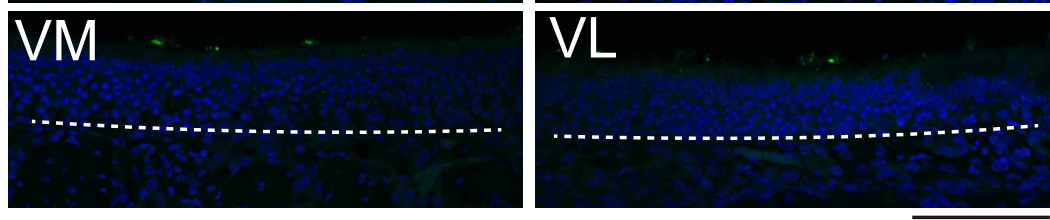

B

SARS-CoV-2

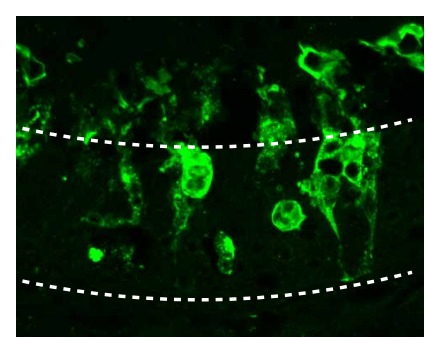

DAPI

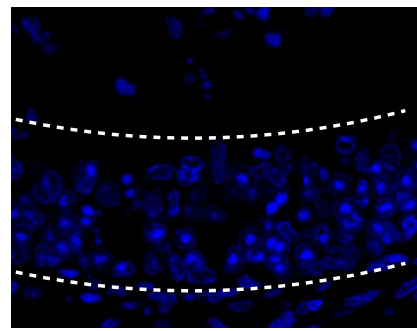

MERGE

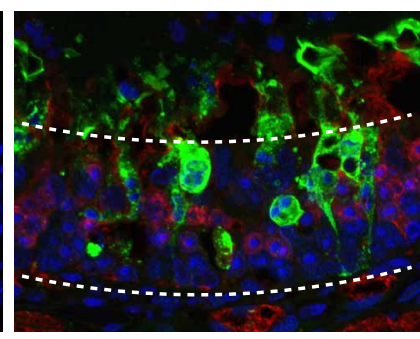

VL
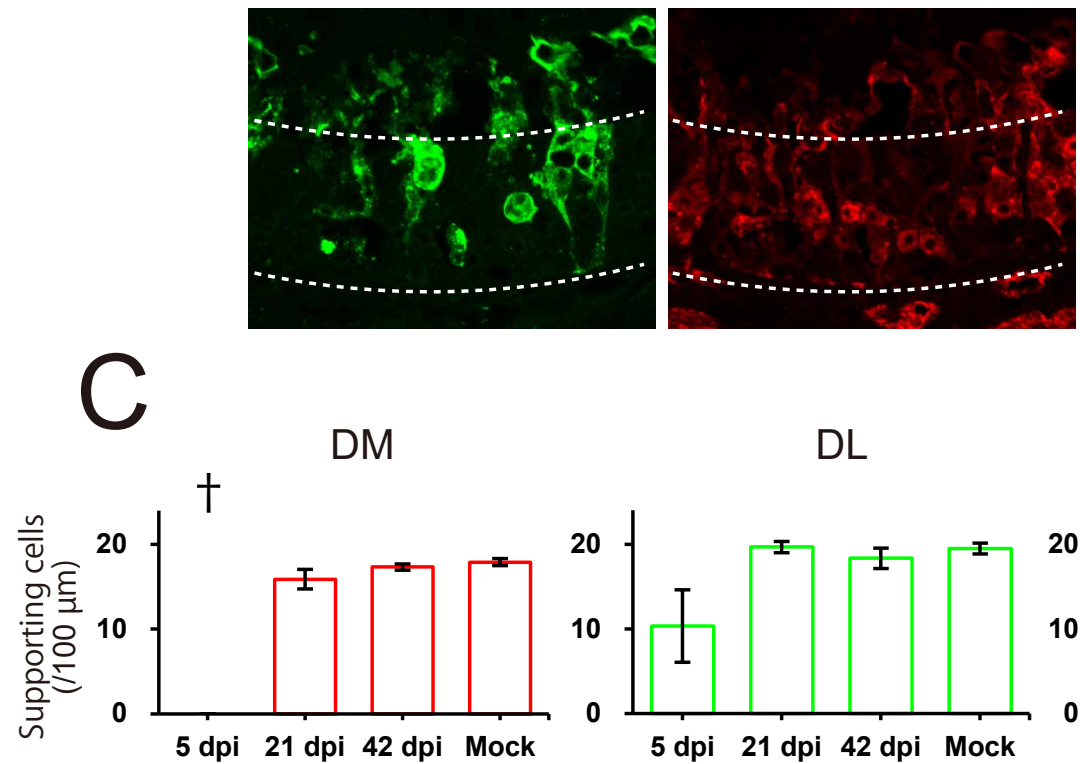

VM

*
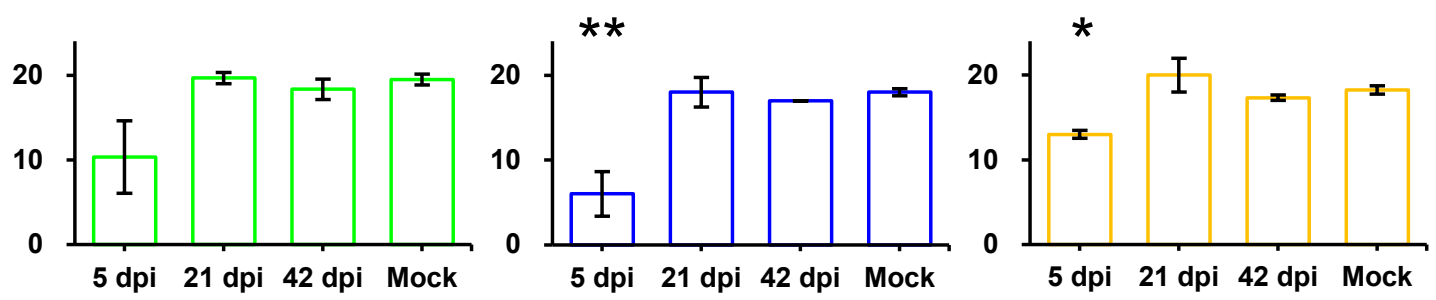
A

\section{OMP NQ01 DAPI}

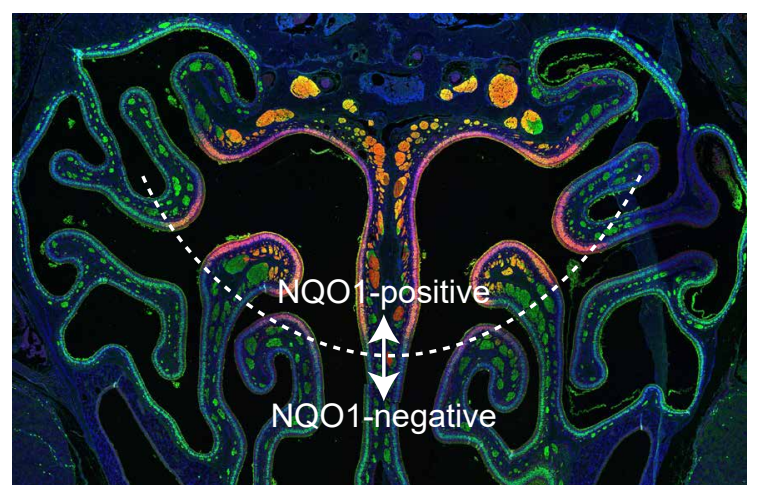

C

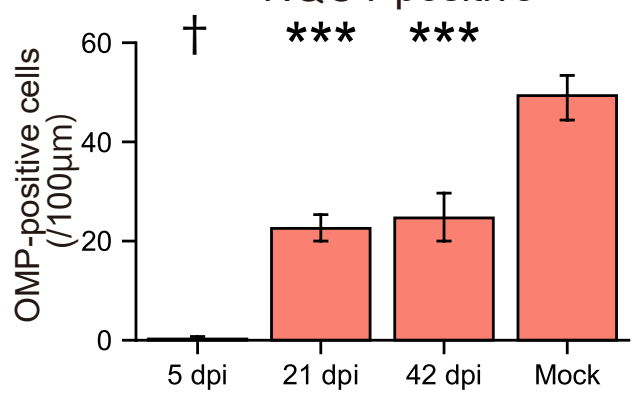

$E$

Iba1 NQ01 DAPI

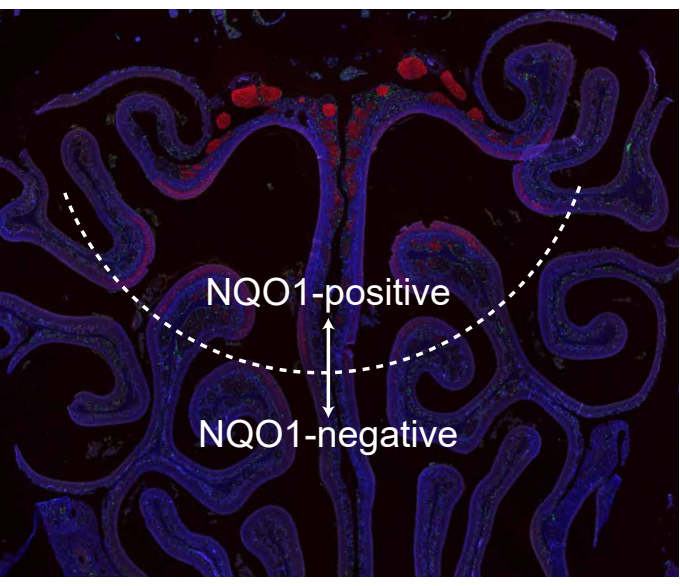

G

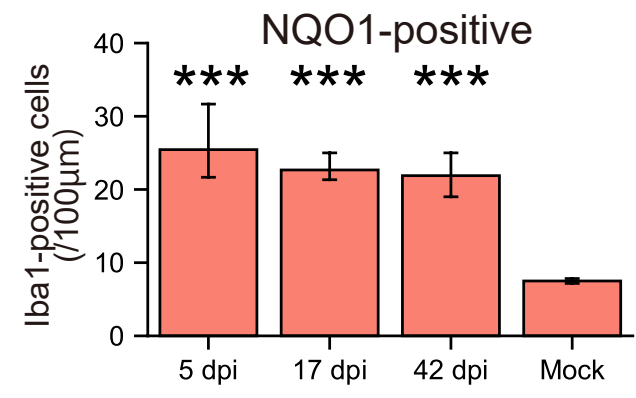

F
B

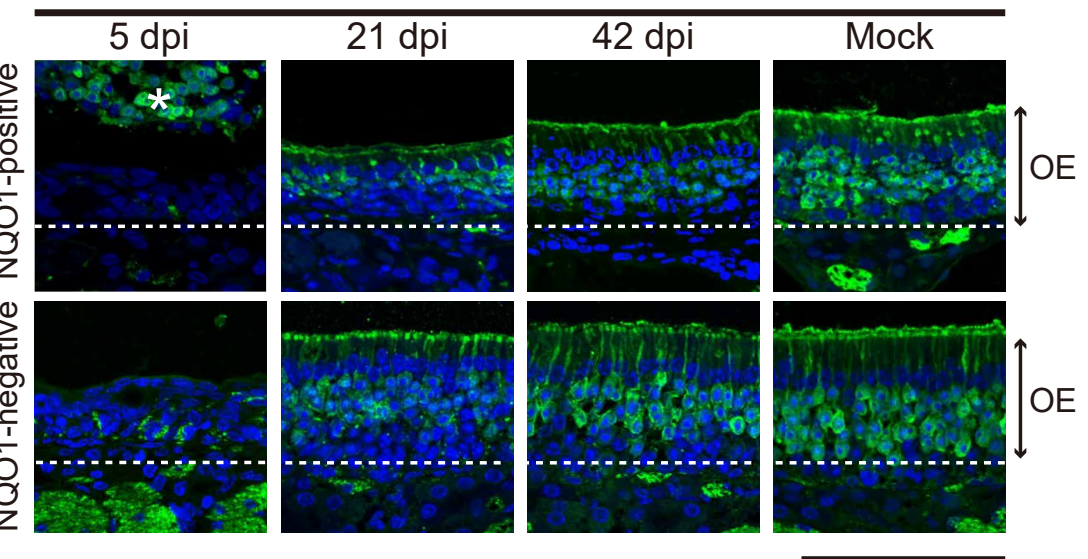

NQO1-negative
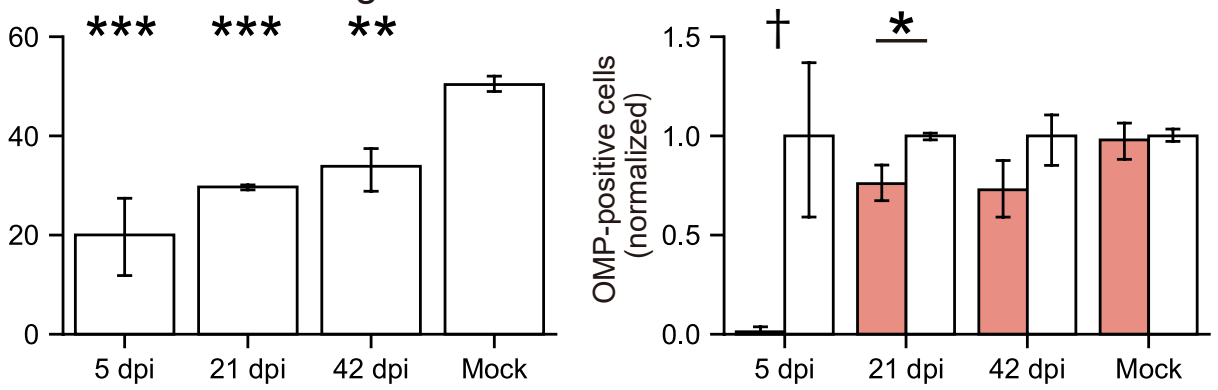

Iba1 DAPI
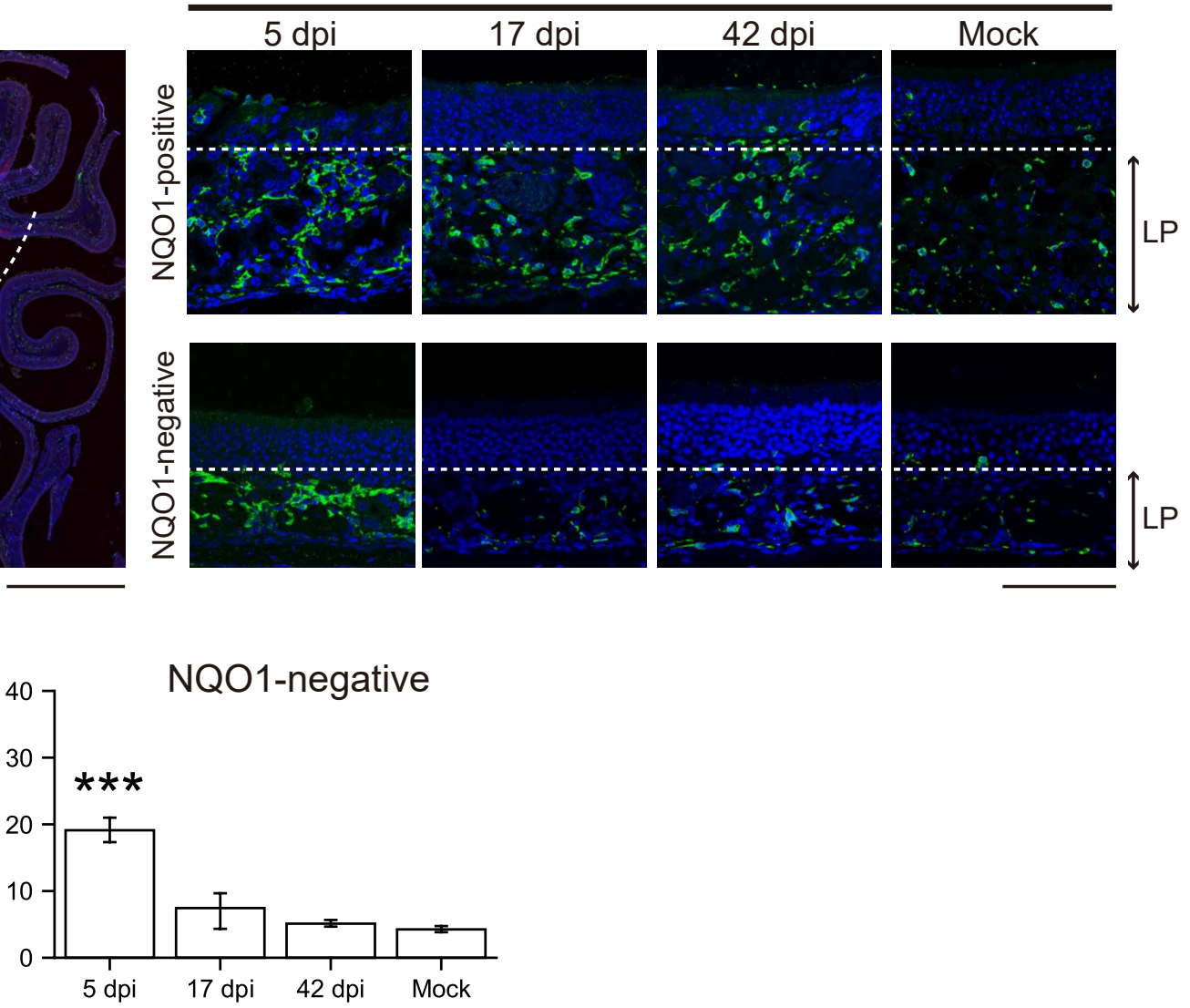


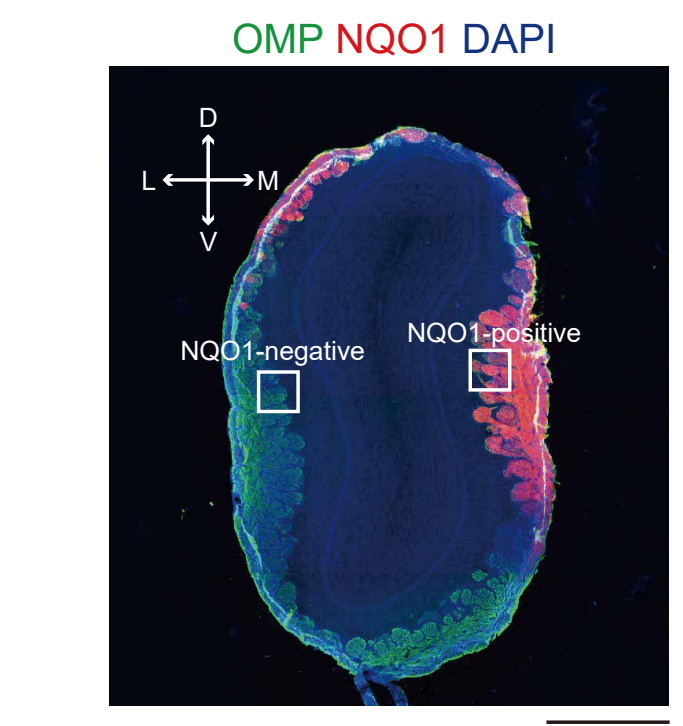

C

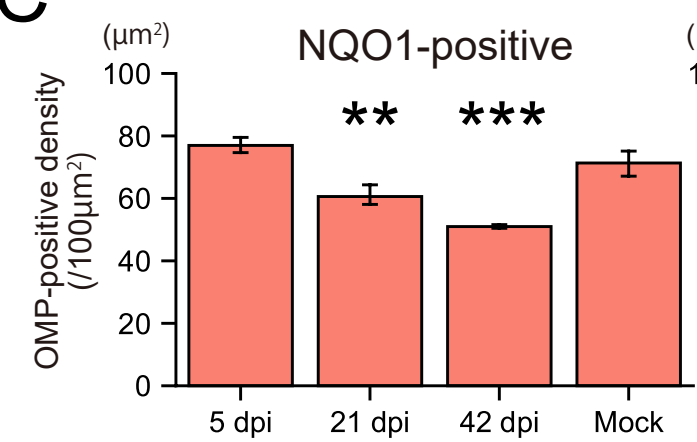

E available under aCC-BY 4.0 International license.

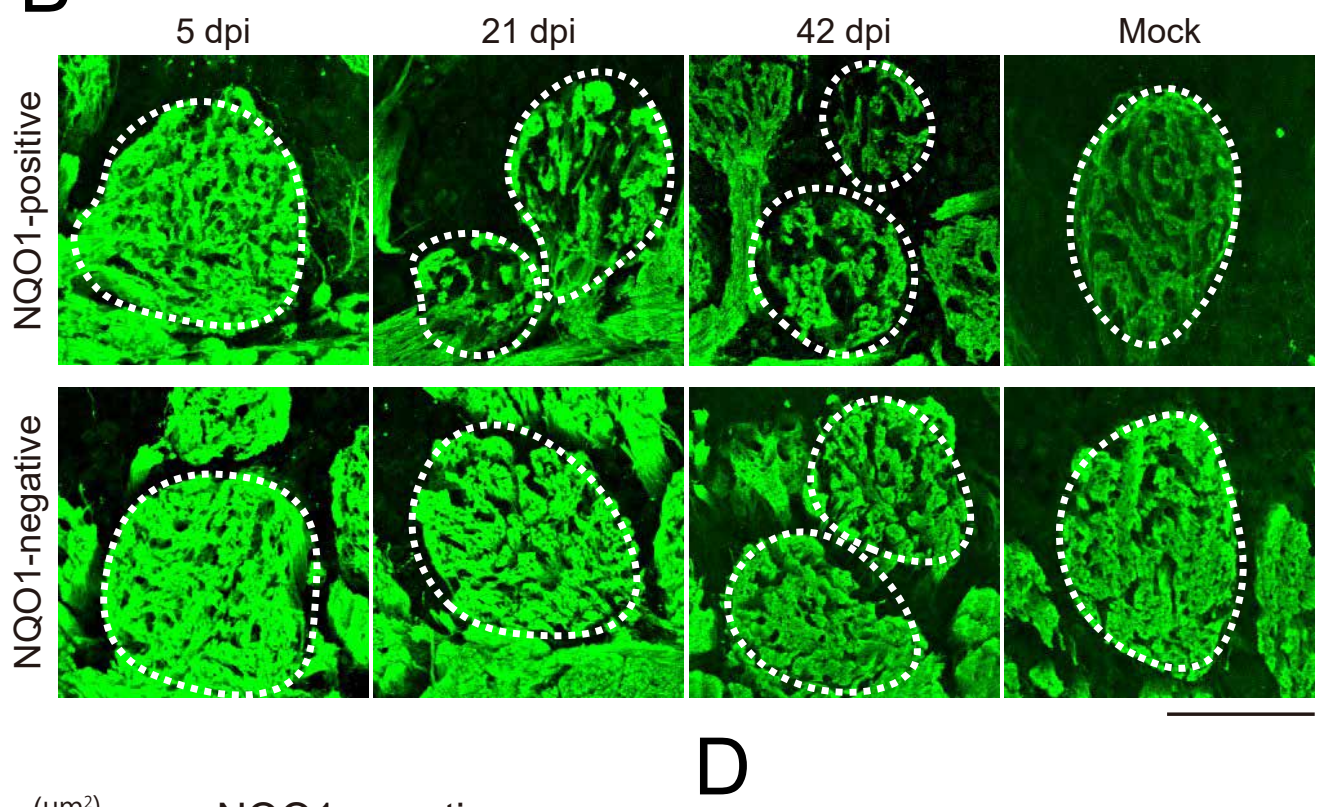

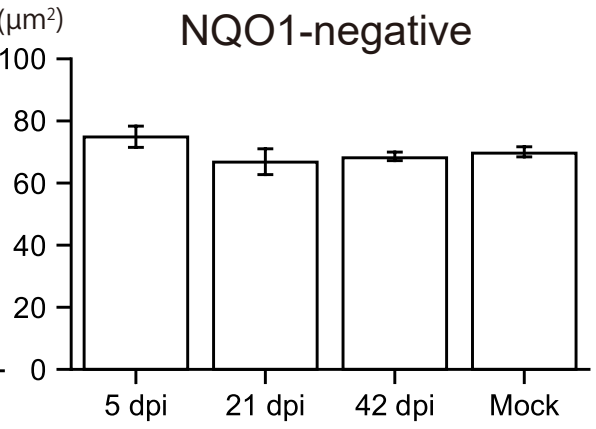

NQO1-positive

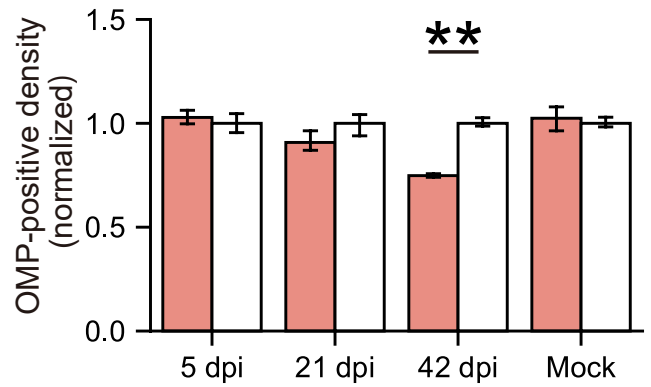

NQO1-negative
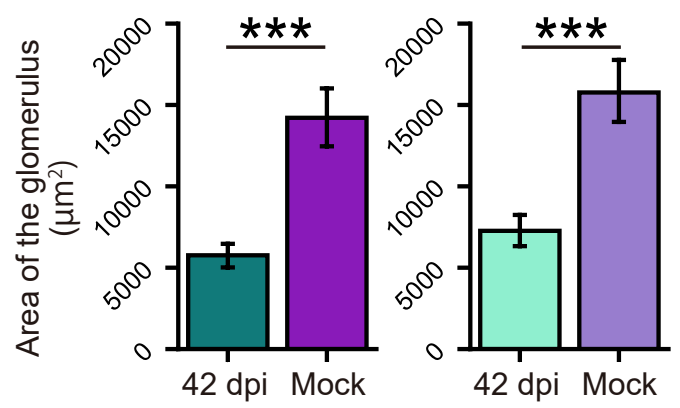

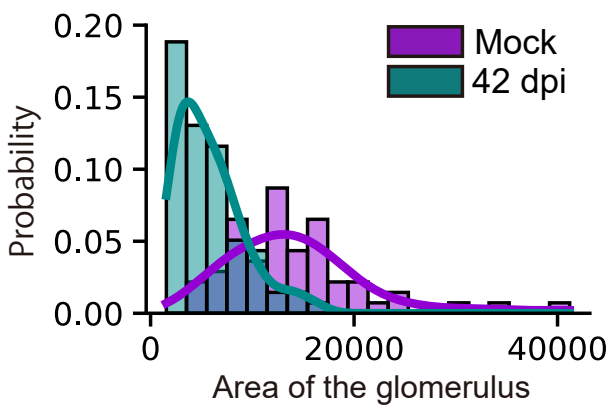

$\left(\mu \mathrm{m}^{2}\right)$

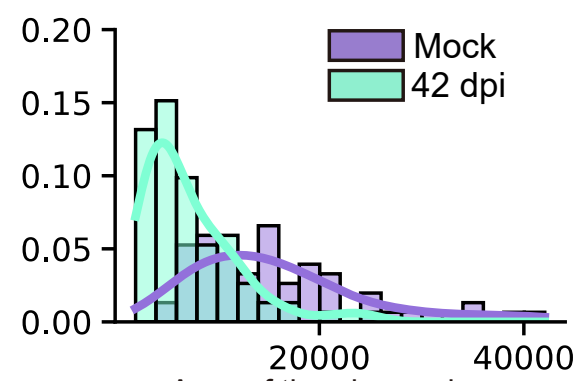

Area of the glomerulus

$\left(\mu \mathrm{m}^{2}\right)$ 

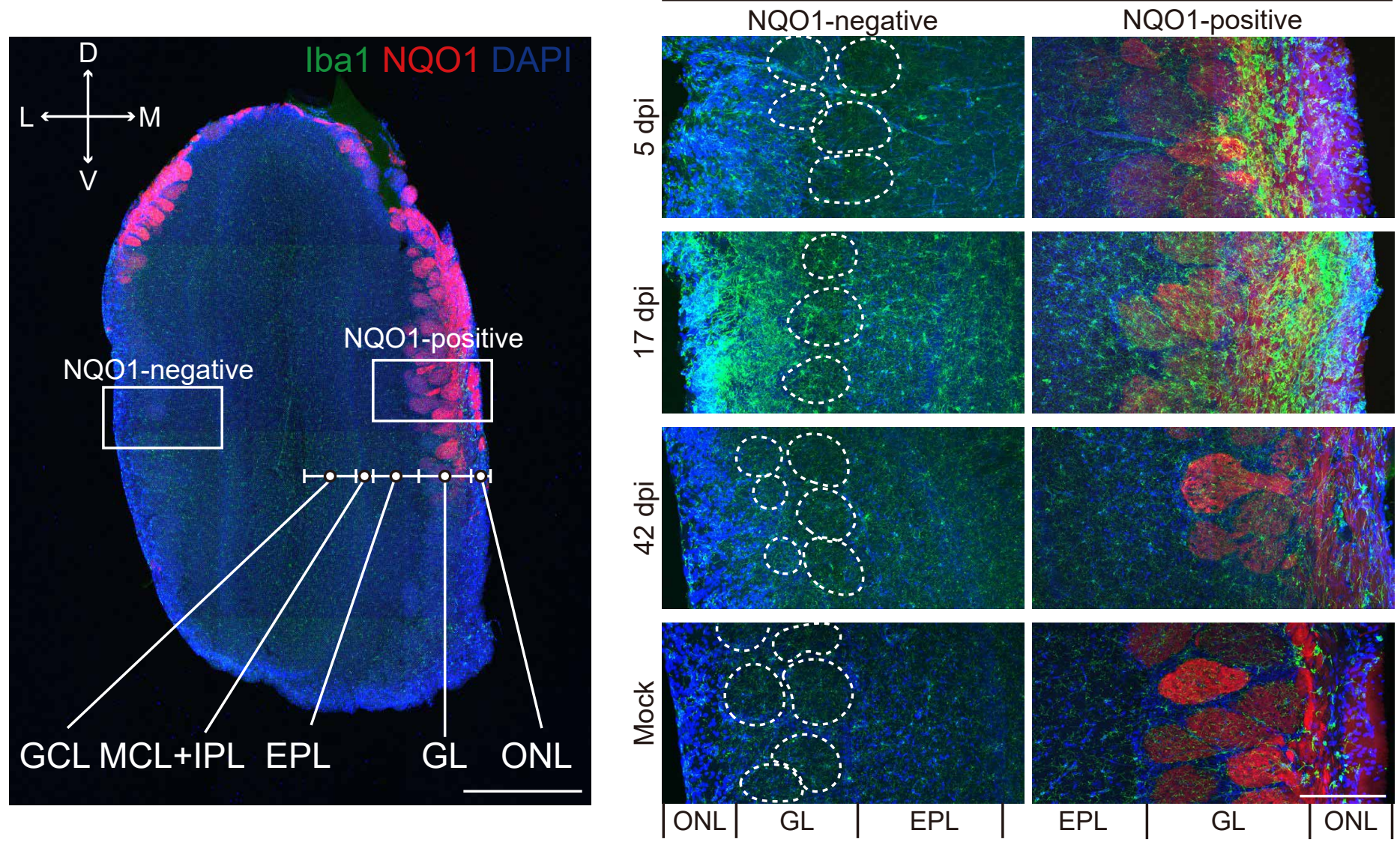

C
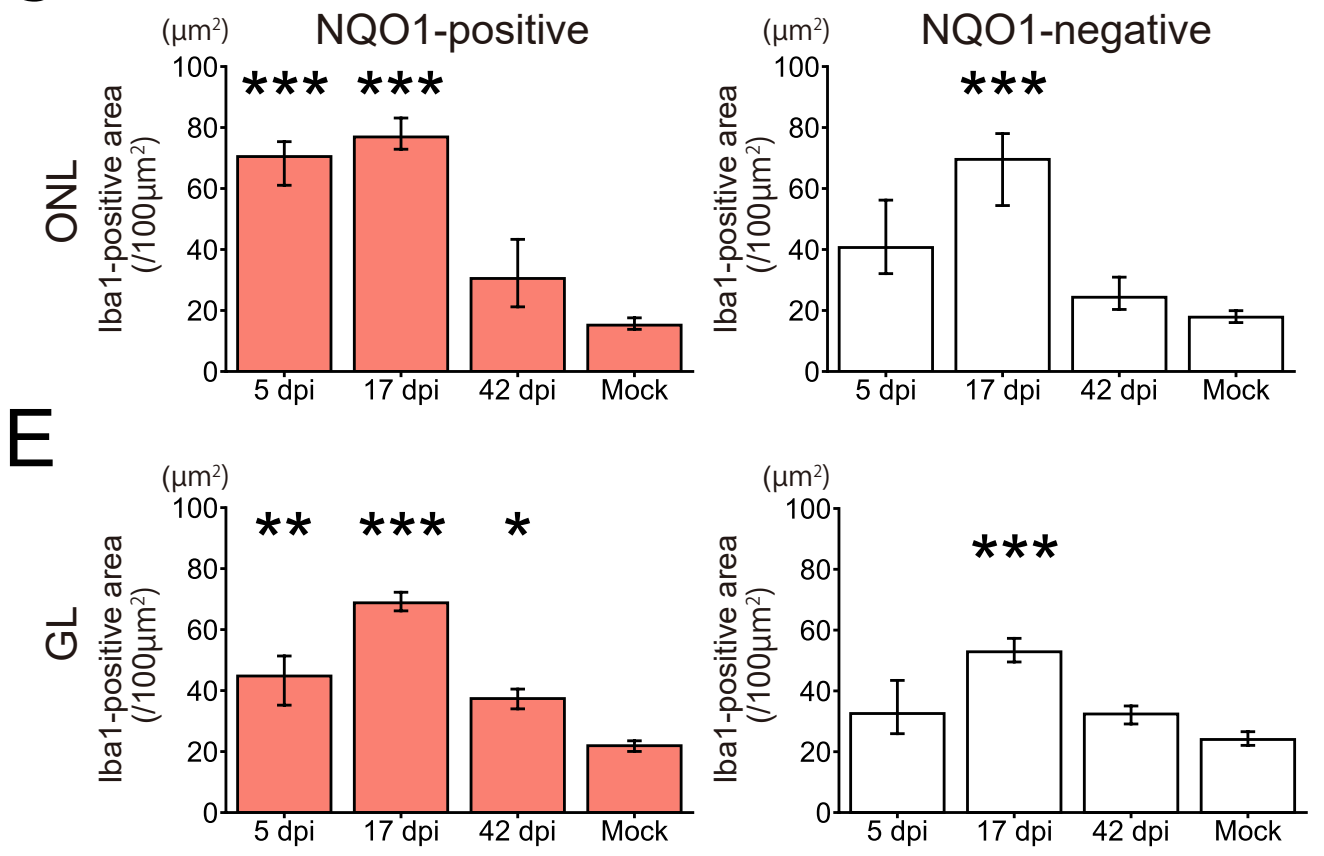

G
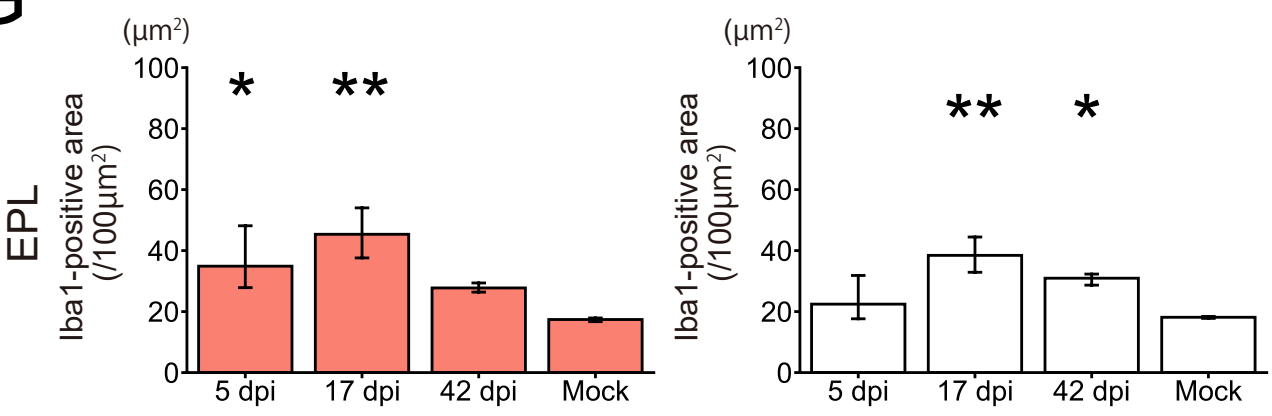
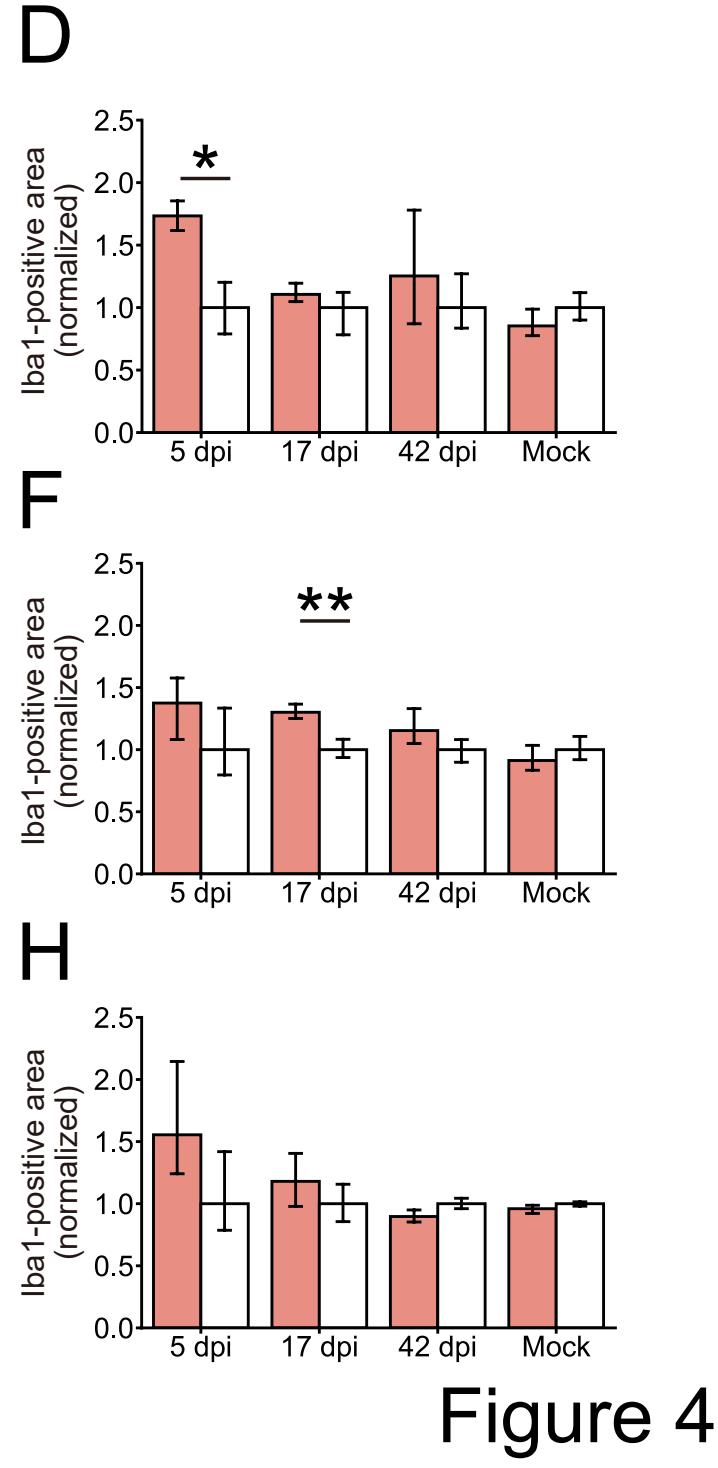
bioRxiv preprint doi: https://doi.org/10.1101/2021.11.04.467274; this version posted November 5, 2021. The copyright holder for this preprint A (which was not certified by peer revievtsis the author/funder, who has granted bioRxiv a license to display the preprint in perpetuity. It is made
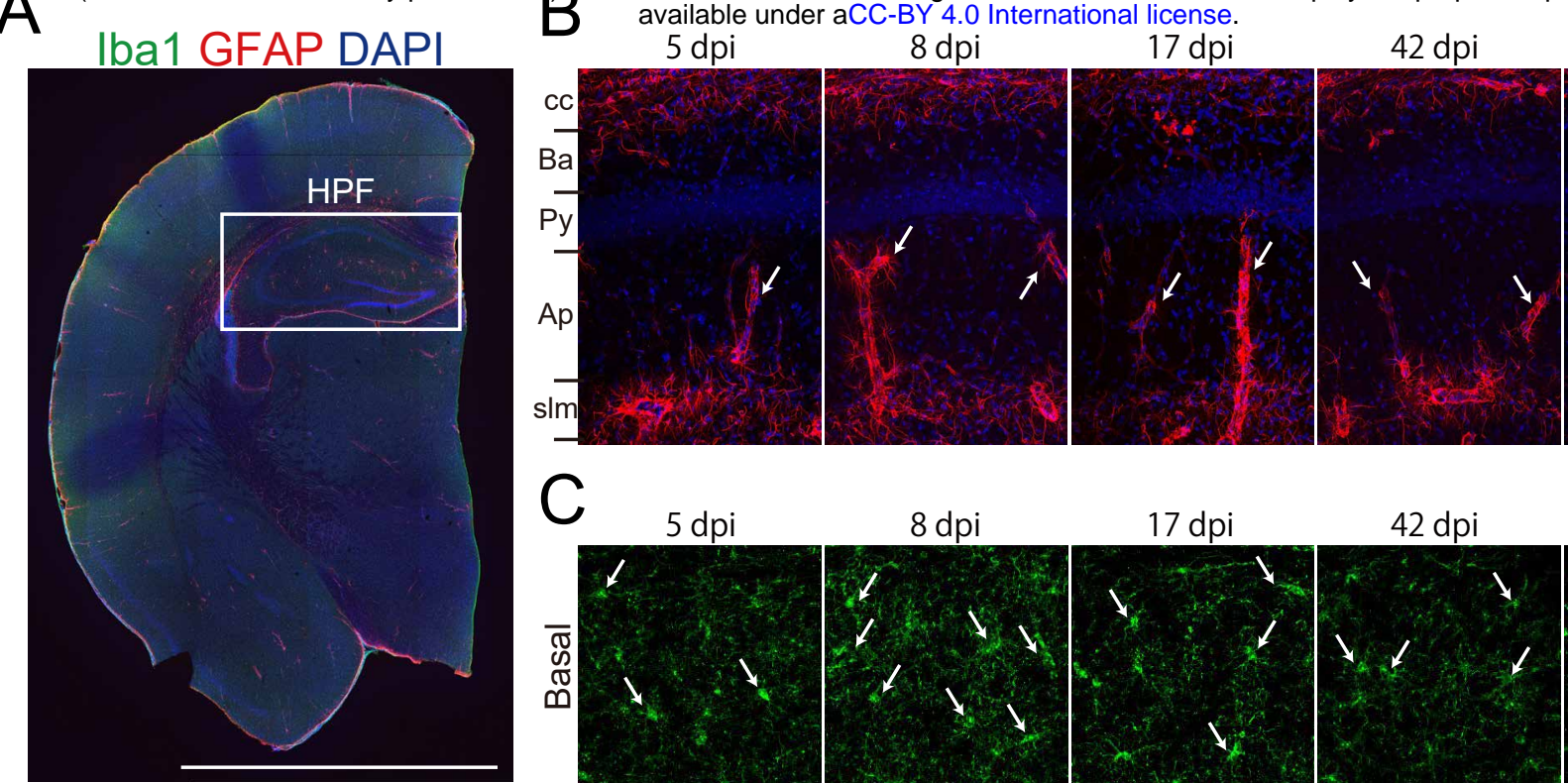

Mock

C
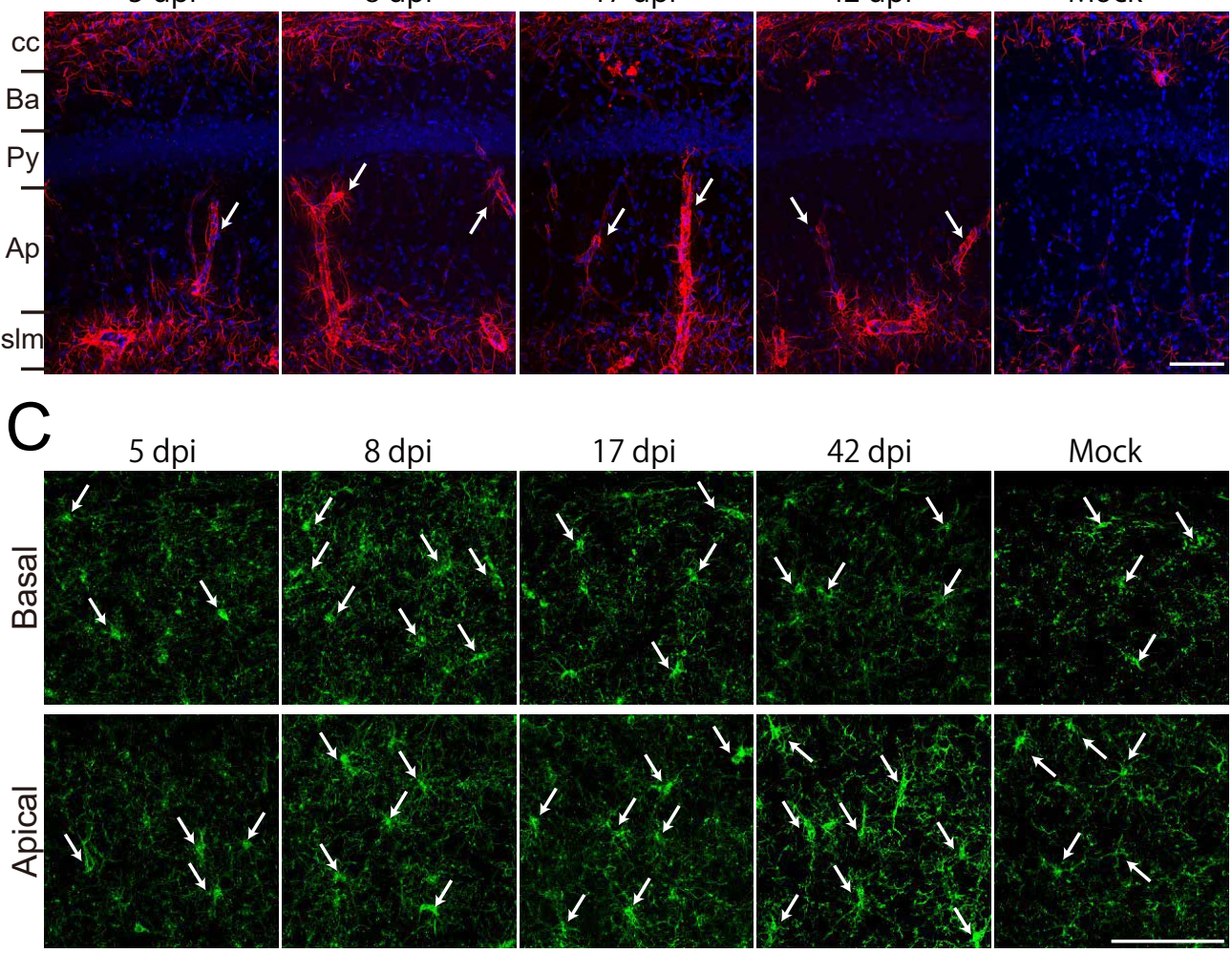

Mock

D

Apical
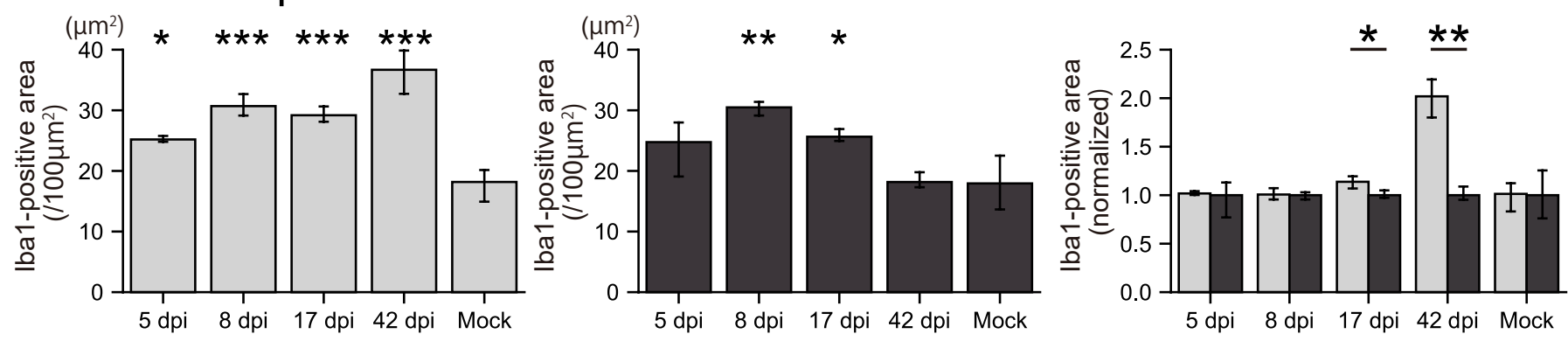

F

G

$\mathrm{H}$
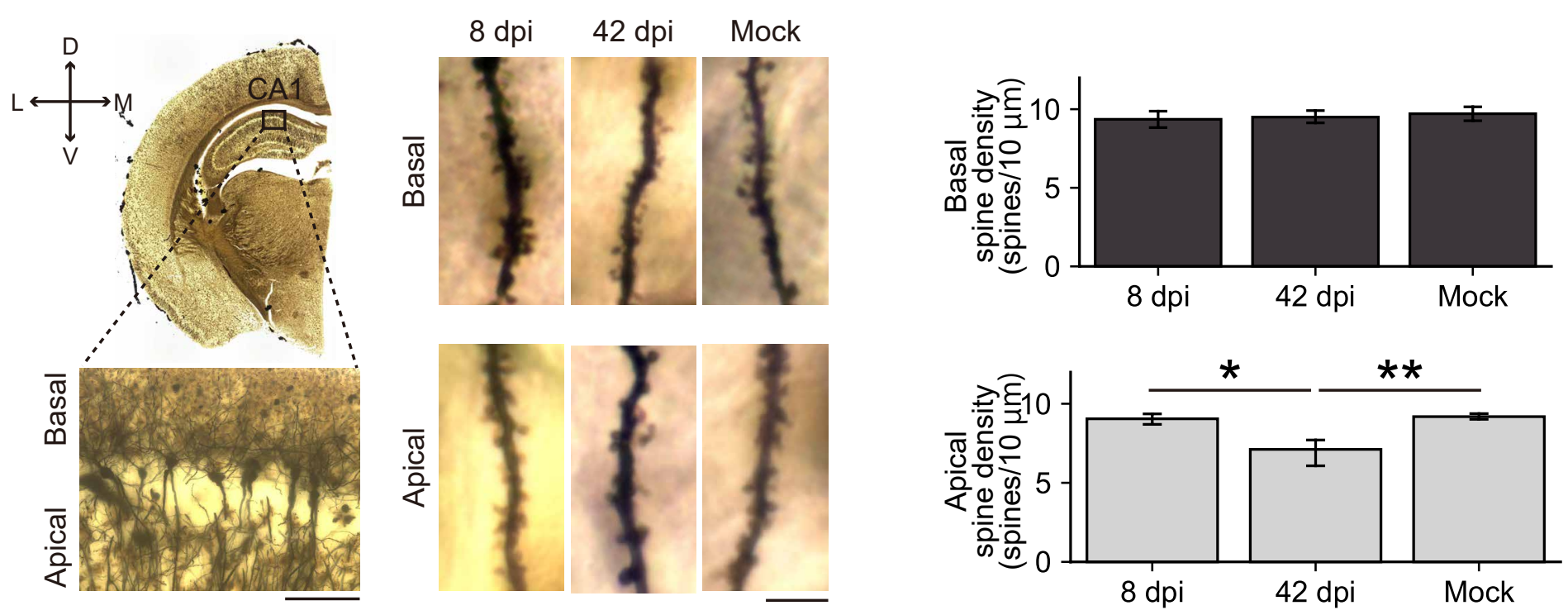

Figure 5 
bioRxiv preprint doi: https://doi.org/10.1101/2021.11.04.467274; this version posted November 5, 2021. The copyright holder for this preprint (which was not certified by peer review) is the author/funder, who has granted bioRxiv a license to display the preprint in perpetuity. It is made A available under aCC-BY 4.0 International license.

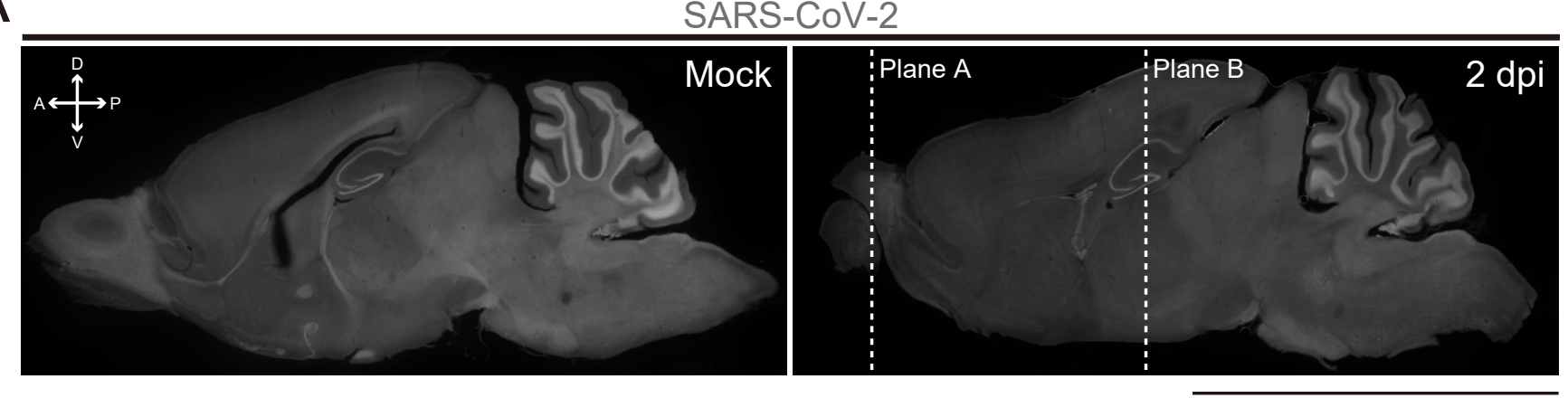

B SARS-CoV-2 NQO1 DAPI

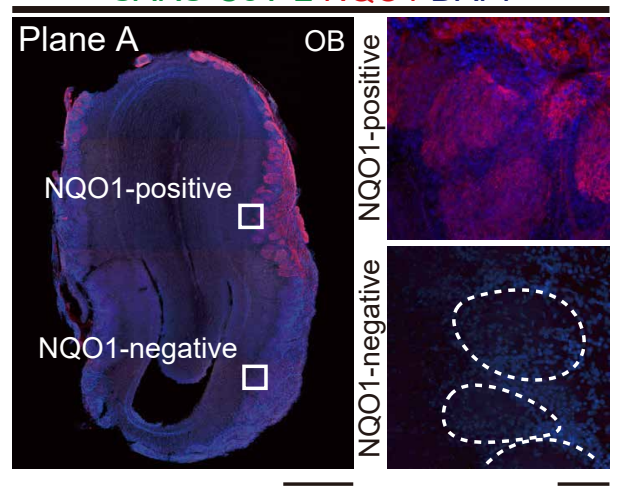

C

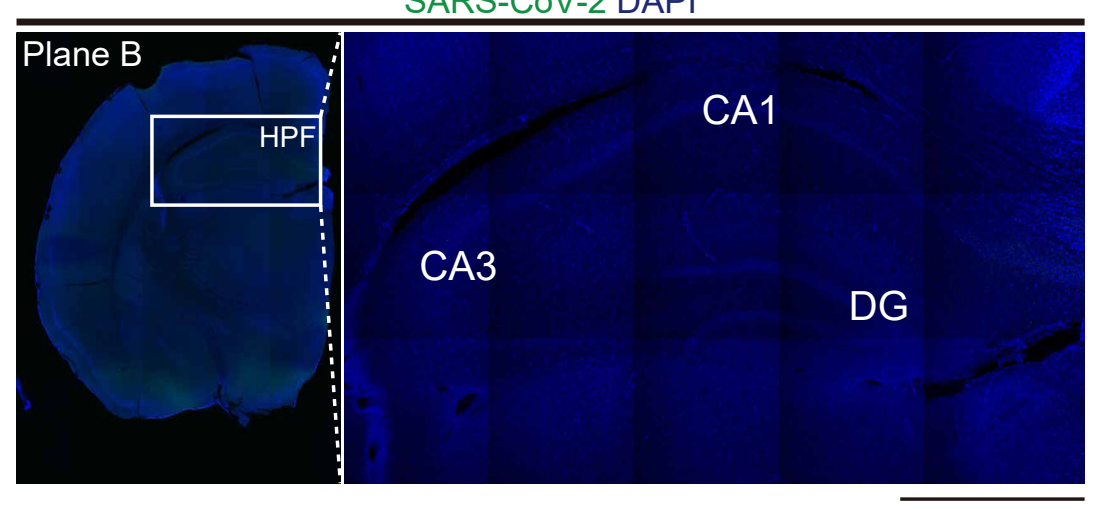



A

bioRxiv preprint doi: https:/doi.org/10,1101/2021.11.04 467274; this version posted November 5, 2021. The copyright holder for this preprint (which was not certified by peer review) is the author/funder, who has granted bioRxiv a license to display the preprint in perpetuity. It is made

Mock

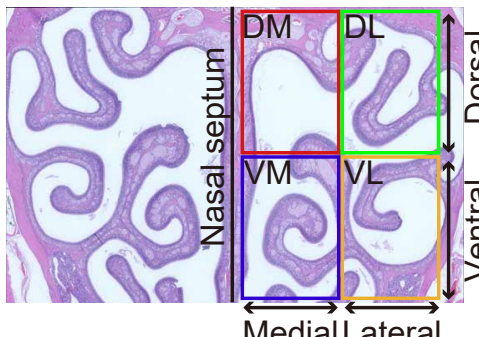

21 dpi

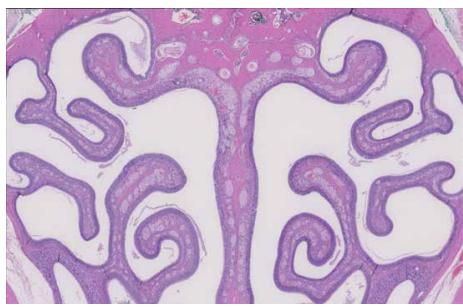

B

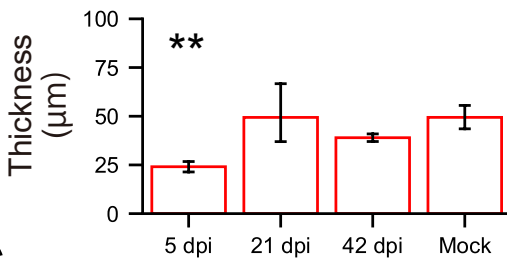
available under aCC-BY 4.0 International license.

ste
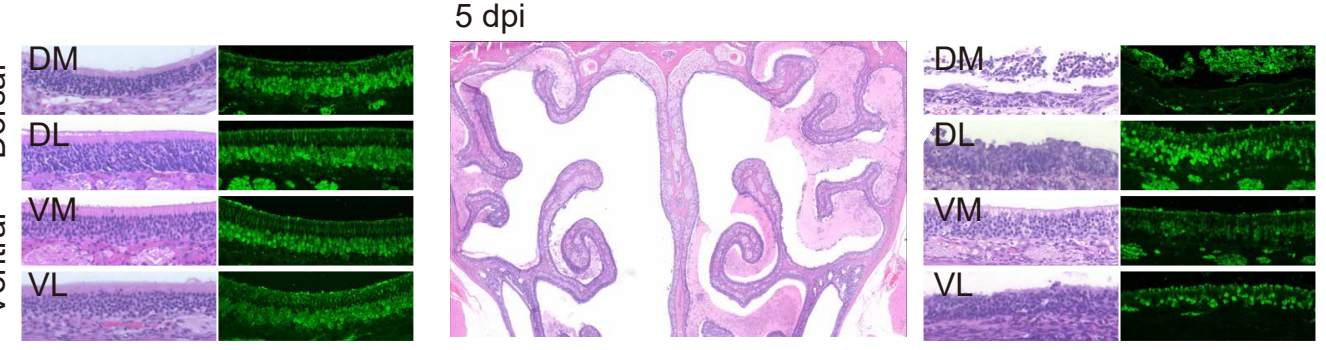

\section{$42 \mathrm{dpi}$}

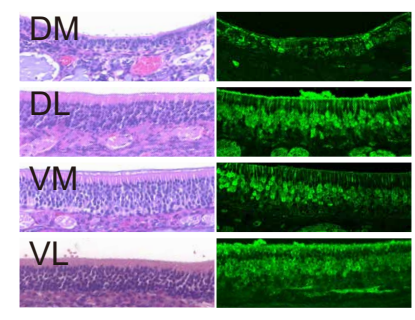

DL
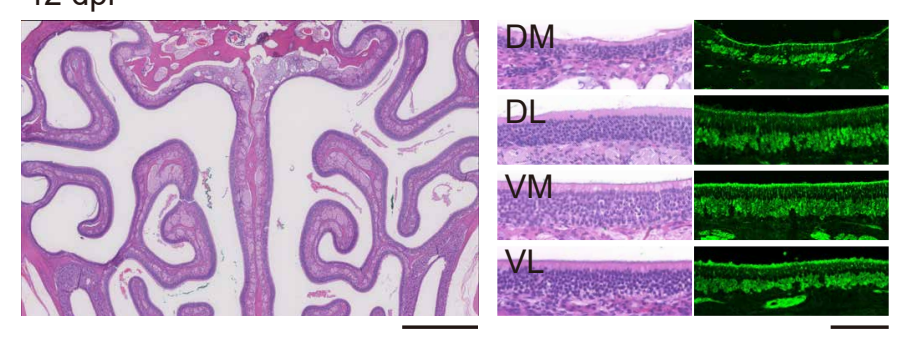

VM

VL
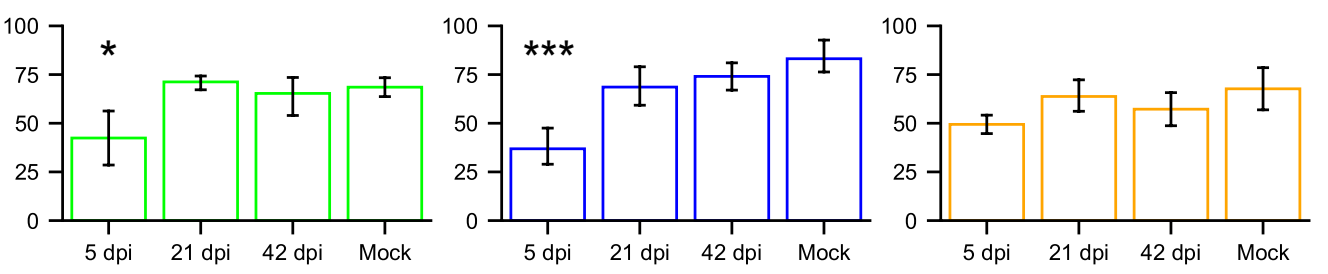
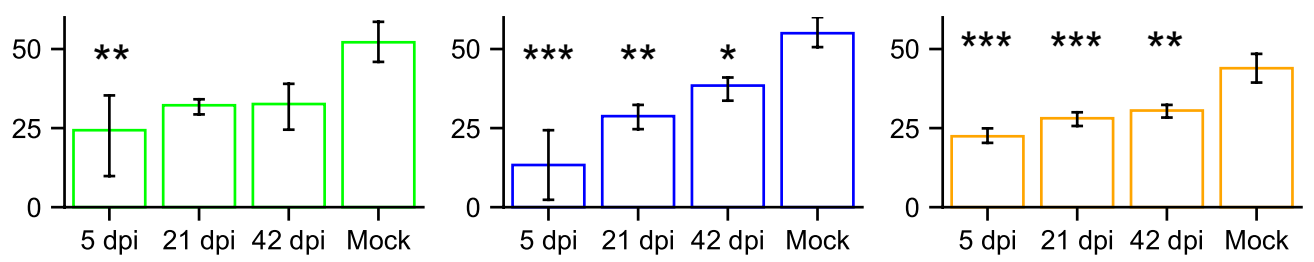

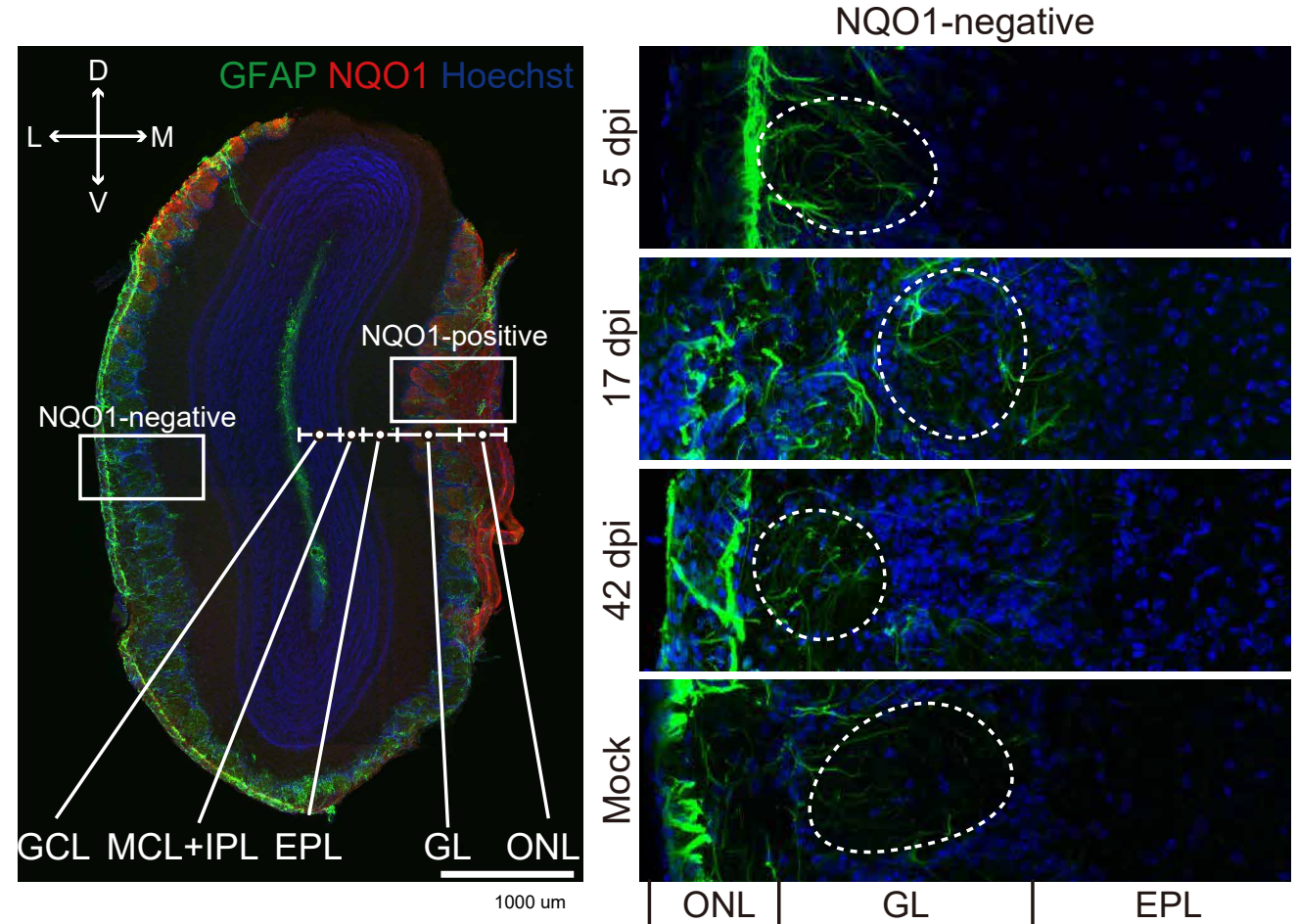

\begin{tabular}{l|l|l} 
ONL & GL & EPL
\end{tabular}
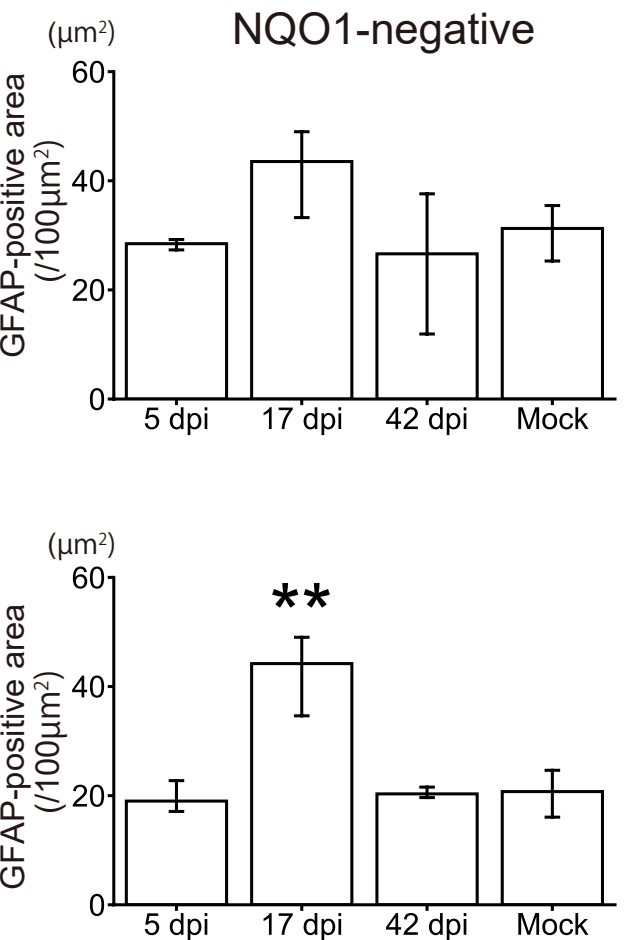
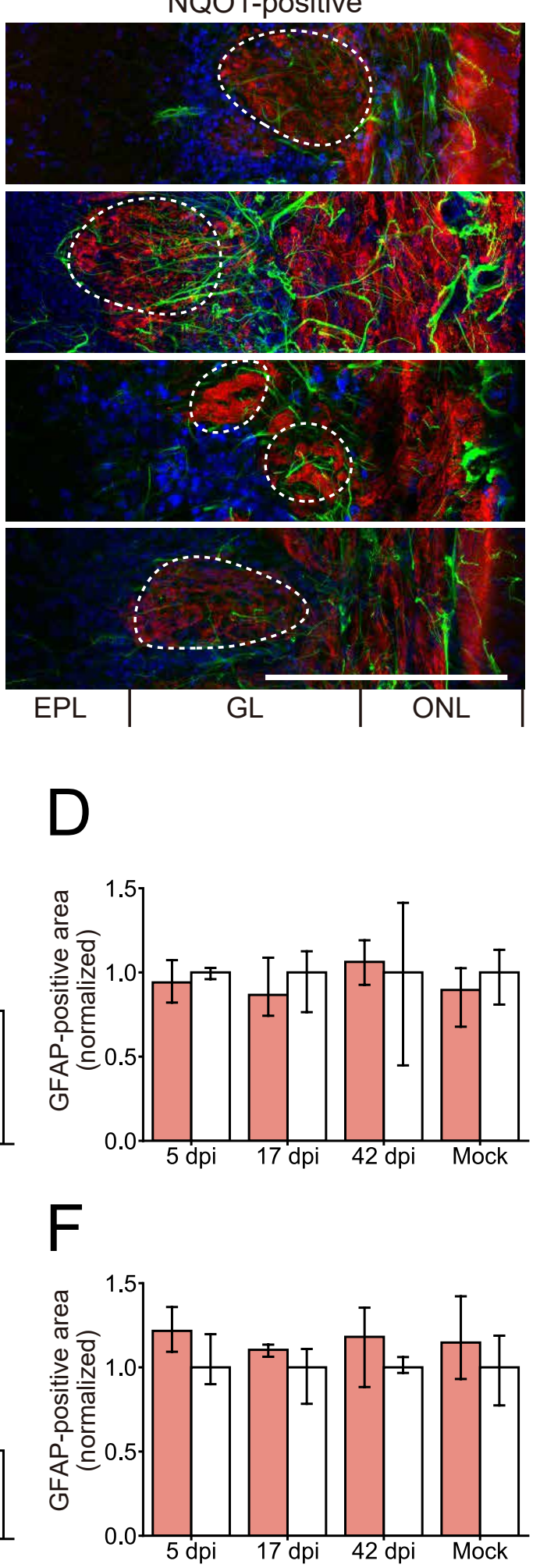
bioRxiv preprint doi: https://doi.org $1101 / 2021.11 .04 .467274$; this version posted November 5 , 2021. The copyright holder for this preprint (which was not certified by peer reve $y$ ) is the author/funder, who has granted bjoRxiv a licepse fo display the preprint in perpetuity. It is made available under aCC-BY 4 |domer hatoftlisense. HOeChSt

Iba1 GFAP Hoechst
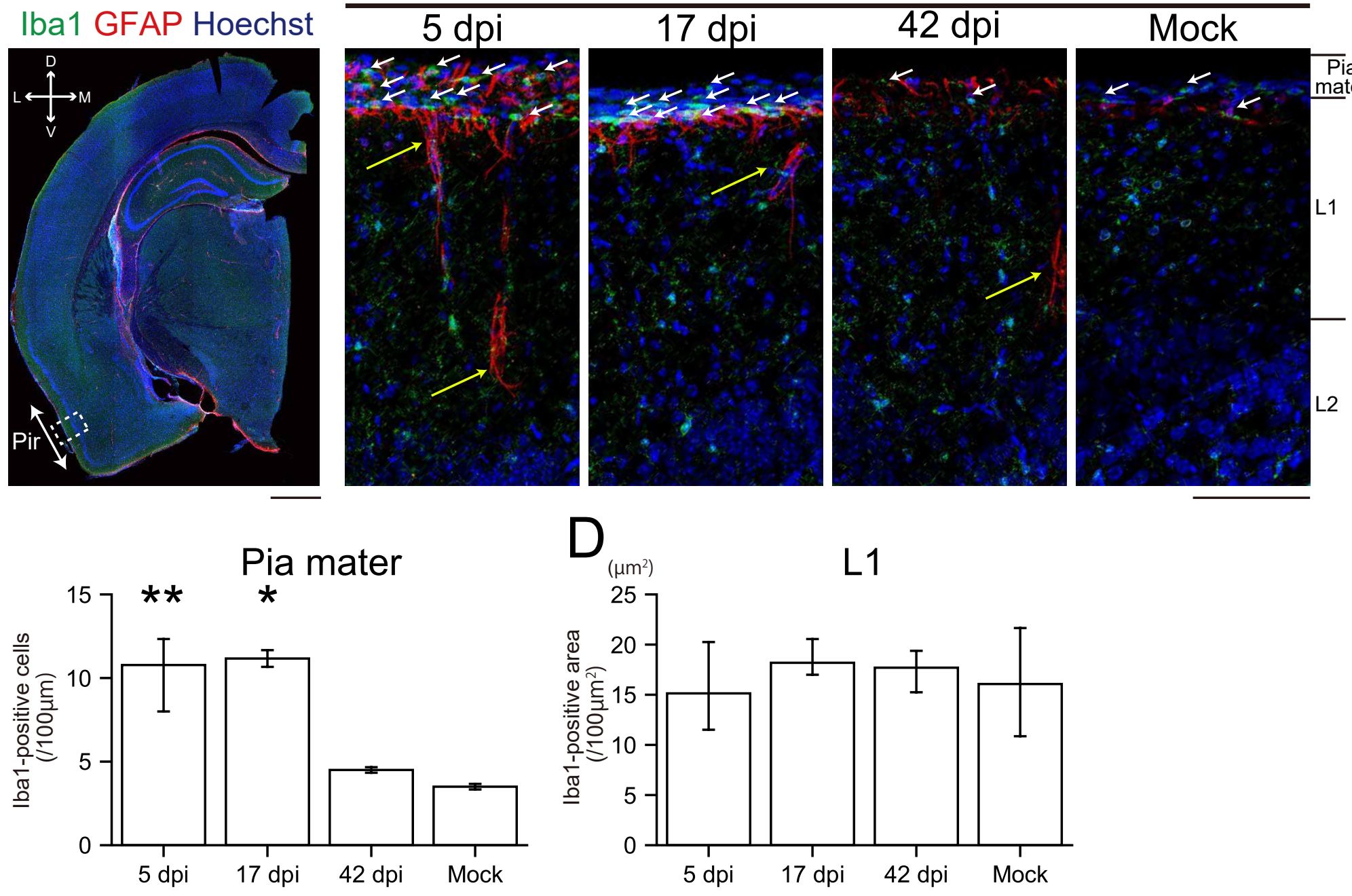
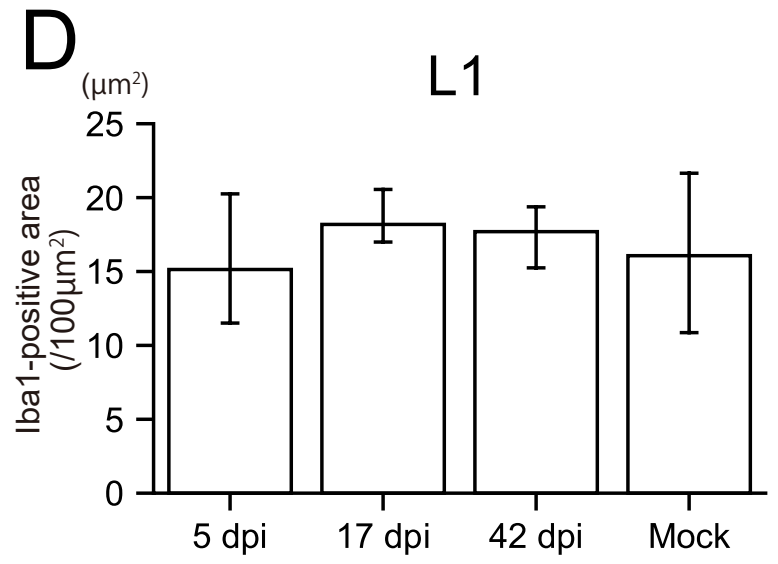